\title{
New Methods for Computing Power System Dynamic Response for Real-Time Transient Stability Prediction
}

\author{
Chih-Wen Liu, Member, IEEE and James S. Thorp, Fellow, IEEE
}

\begin{abstract}
Using new systems capable of making synchronized phasor measurements, the real-time stability assessment of a transient event in power systems has become an important area of investigation. Using these phasor measurements as input conditions for computing a relatively good, simplified dynamic model can yield accurate and real-time transient stability prediction in a central location equipped with high-speed computers. In an effort to reduce the computing time for integrating the differential/aAlgebraic equation (DAE) model of postfault power system dynamics for prediction use, this paper presents a faster Implicitly Decoupled PQ Integration technique. Two piecewise dynamic equivalents are also proposed, i.e., piecewise constant current load equivalent and piecewise constant transfer admittance equivalent. These equivalents can eliminate the algebraic equations by approximating the load flow solution piecewisely such that only internal generator buses are preserved, while approximately retaining the characteristics of the nonlinear loads. The proposed techniques have been tested on two sample power systems with promising simulation results.
\end{abstract}

Index Terms-Decoupled integration, dynamic response, piecewise equivalent, phasor measurements, transient stability.

\section{INTRODUCTION}

W ITH the advent of phasor measurement units (PMU's) [1]-[4] capable of tracking the dynamics of an electric power system, together with modern telecommunication abilities, utilities are becoming able to respond intelligently to an actual event in progress. By the synchronization of sampling with microprocessor based systems, phasor calculations can be placed on a common time scale [5]. Commercially available systems based on Global Positioning System (GPS) satellite time transmissions can provide synchronization to $1 \mu \mathrm{s}$ accuracy, which means that a relative phase angle can be measured to a precision of 0.02 electrical degrees. Utility experience indicates that communication systems can transmit these timetagged phasor measurements to a central location every five cycles [6]. The magnitudes and angles of these phasors indicate the state of a power system, and are used in state estimation and transient stability analysis [7].

The power system itself provides us with the system trajectory up to the current time. The time track of state variables and several of their derivatives are available in an observation window (Fig. 1). Thus, it seems possible that the outcome for a

Manuscript received April 7, 1997; revised September 2, 1998 and June 13, 1999. This paper was recommended by Associate Editor A. Ioinovici.

C.-W. Liu is with the Department of Electrical Engineering, National Taiwan University, Taipei, Taiwan (e-mail: cwliu@cc.ee.ntu.edu.tw).

J. S. Thorp is with the School of Electrical Engineering, Cornell University, Ithaca, NY 14850 USA (e-mail: thorp@ gonzo.ee.cornell.edu).

Publisher Item Identifier S 1057-7122(00)02310-2.

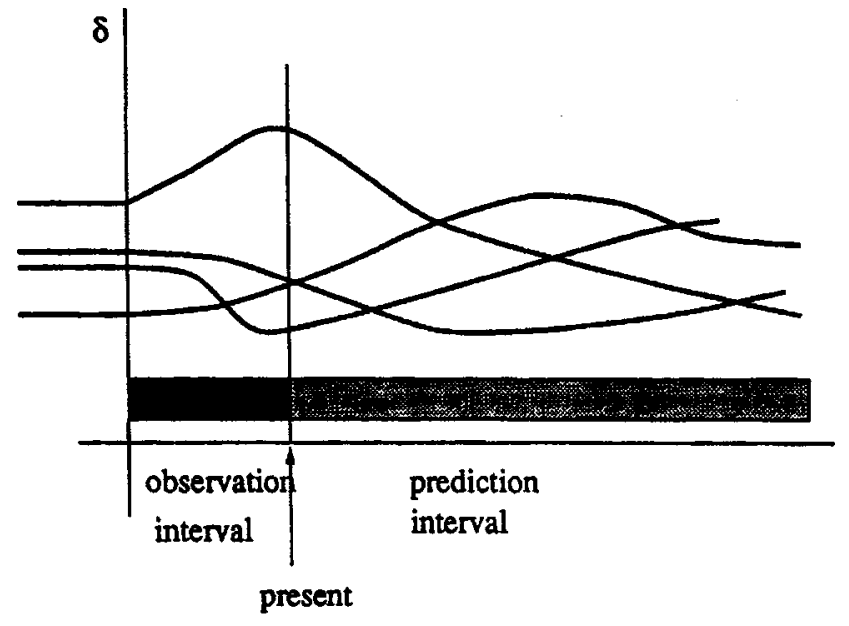

Fig. 1. Prediction of power system stability for adaptive protection.

future time interval of an evolving swing can be calculated with relatively accurate simplified dynamic models. If the outcome of a developing swing can be predicted in real time, it is possible to initiate appropriate protection and control actions, such as out-of-step blocking and tripping, or fast-valve control of turbines.

One such real-time control strategy is already being implemented at the Florida-Georgia interface [8]. An important feature of the Florida-Georgia situation is that interarea oscillations between the two regions can always be modeled as a simplified two-machine equivalent system. When interarea oscillations occur, phasor measurements are taken within Florida and Georgia in order to develop the corresponding state of the two-machine equivalent. Future stability is then determined by applying the equal area criterion. This method of prediction is used for adaptive out-of-step relaying at the Florida-Georgia interface.

Many existing transient stability assessment techniques, although simple in off-line application, are too complicated for real-time prediction use. However, real-time monitoring obviates the need for some of these techniques since the system itself is actually solving the differential/algebraic equations (DAE's). What is required is a computationally efficient way of processing real-time measurements to determine whether an evolving event will ultimately be stable or unstable. The problem of real-time stability prediction for a simplified model can be answered with powerful workstations in control centers, which enable one to integrate the system trajectory in real time. Given a simplified model and an initial condition (observed phasor measurements), a workstation takes just a 
few hundredths of a second to compute a system trajectory one second into the future.

In an effort to reduce the computing time for integrating a DAE description of a postfault dynamic model, this paper presents an efficient implicitly decoupled PQ integration (decoupled integration) technique. While DAE description is accurate for an adopted model, it becomes complex as the size of a study system increases. Therefore, two piecewise dynamic equivalents are proposed, i.e., the piecewise constant current load equivalent (current load equivalent) and piecewise constant transfer admittance equivalent (admittance equivalent). Together, these can eliminate the algebraic equations by approximating the load flow solution piecewisely so that only internal generator buses are preserved, while retaining the approximate characteristics of nonlinear loads.

\section{ReAl-Time Prediction Problem AND DynAmic Model}

Our research addresses the question of accomplishing stability prediction when the system does not always reduce to a previously known two-machine equivalent. Possible methods of approaching this problem which we have investigated fall into two broad categories.

1) Infer a small-size (e.g., two-, three-, or four-machine) equivalent from the postfault phasor measurements, which can model the particular oscillation mode of the fault in progress. Solve the model forward in time in order to predict future behavior.

2) Use an appropriately simplified postfault dynamic model of the system (e.g., the 39 bus model for New England) which adequately covers the many modes of oscillation initiated by different events. Solve the model faster than real time if computational resources permit, or else train a pattern recognition tool (e.g., decision trees and neural networks) off-line in order to associate in real-time the postfault phasor measurements with the outcome of future behavior.

Previously, in [9]-[11] short-term prediction algorithms for the above two strategies were proposed. In this paper, we propose new methods to solve the dynamic model faster than real time to enhance the second strategy.

An appropriately simplified postfault dynamic model plays a critical role in real-time stability prediction problem. A detailed model, although more accurate for prediction of a transient event over a longer window may involve so much computation such that it cannot meet real-time demand. On the other hand, an overly simplified model, such as the classical model of a generator with a constant impedance load model, has been observed to give overly optimistic stability results [12]. In a real-time stability prediction problem, a model which gives more conservative results is preferable to a model which gives more optimistic results. This is because failure to execute necessary special protection schemes in real time can prove quite costly [13]. It seems that the classical model of generator with static composite constant impedance, constant current and constant power (referred to as ZIP) load model offers an effective compromise between accuracy and simplicity required for real-time applications [12]. Therefore, in our study, we adopt the classical model of generator with ZIP load model to describe the dynamics of a postfault power system. We first derive the DAE description for the adopted model, and then derive piecewise dynamic equivalents.

\section{A. Differential/Algebraic Equation Description}

Suppose there are $\mathbf{N}_{\mathbf{g}}$ generators and $\mathbf{N}_{\mathbf{0}}$ buses connected by transmission lines and transformers, with $\mathbf{N}_{\mathbf{0}}-\mathbf{N}_{\mathrm{g}}$ buses having loads and no generations. It is convenient to introduce fictitious buses representing the internal generation voltages. These fictitious buses are connected to the generator buses via reactances accounting for transient reactances and connecting lines. Thus the total number of buses in the augmented system is $N_{0}+N_{g}=N$ buses. We order the buses as follows:

$$
\begin{array}{ll}
\left(1,2, \ldots, \mathbf{N}_{\mathbf{g}}\right) & \text { generator internal buses } \\
\left(\mathbf{N}_{\mathbf{g}}+1, \ldots, 2 \mathbf{N}_{\mathbf{g}}\right) & \text { generator terminal buses } \\
\left(2 \mathbf{N}_{\mathbf{g}}+1, \ldots, \mathbf{N}\right) & \text { load buses. }
\end{array}
$$

A quasi-static network/load representation is assumed. Let the complex voltage at $\mathbf{i t h}$ bus be the phasor $\mathbf{E}_{\mathbf{i}}=\mathbf{V}_{\mathbf{i}} \angle \delta_{\mathbf{i}}$, where $\delta_{\mathbf{i}}$ is the bus phase angle with respect to a synchronously rotating reference frame. The ith generator frequency deviation is denoted as $\omega_{\mathbf{i}} \doteq \dot{\delta}_{\mathbf{i}}$. The network is represented by the node (bus) admittance matrix $\mathbf{Y}=\mathbf{G}+\mathbf{j B}$.

1) Generator Dynamic Equations: We assume that the generators are modeled by constant internal voltage sources behind transient reactance. The angle of this voltage is determined by the swing equation

$$
\begin{gathered}
\dot{\delta}_{\mathbf{i}}=\omega_{i}, \quad i \in 1,2, \ldots, \mathbf{N}_{\mathrm{g}} \\
\mathbf{m}_{i} \dot{\omega}_{i}+\mathbf{d}_{i} \omega_{i}=\mathbf{P}_{\mathbf{m i}}-\mathbf{P}_{i}, \quad i \in 1,2, \ldots, \mathbf{N}_{g}
\end{gathered}
$$

where

$$
\begin{gathered}
\mathbf{m}_{i}>0 \\
\mathbf{d}_{i}>0 \\
\mathbf{P}_{\mathrm{mi}}
\end{gathered} \begin{aligned}
& \text { generator inertial constant } \\
& \text { generator damping coefficient } \\
& \mathbf{P}_{i}=\sum_{j} \mathbf{G}_{i j} \mathbf{V}_{i} \mathbf{V}_{j} \cos \left(\delta_{i}-\delta_{j}\right) \\
& +\sum_{j} \mathbf{B}_{i j} \mathbf{V}_{i} \mathbf{V}_{j} \sin \left(\delta_{i}-\delta_{j}\right), \quad j \in \mathbf{N}_{\mathbf{g}}+1, \ldots, 2 \mathbf{N}_{\mathbf{g}} .
\end{aligned}
$$

2) Transmission System Equations: For a lossy transmission system the power flowing through the transmission lines connected to the $i$ th bus can be written as

$$
\begin{aligned}
\mathbf{P}_{i}= & \sum_{j} \mathbf{G}_{i j} \mathbf{V}_{i} \mathbf{V}_{j} \cos \left(\delta_{i}-\delta_{j}\right) \\
& +\sum_{j} \mathbf{B}_{i j} \mathbf{V}_{i} \mathbf{V}_{j} \sin \left(\delta_{i}-\delta_{j}\right), \quad \mathbf{i}, \mathbf{j} \in \mathbf{N}_{\mathbf{g}}+1, \ldots, \mathbf{N}
\end{aligned}
$$

$$
\begin{aligned}
\mathbf{Q}_{i}= & \sum_{j} \mathbf{G}_{i j} \mathbf{V}_{i} \mathbf{V}_{j} \sin \left(\delta_{i}-\delta_{j}\right) \\
& -\sum_{j} \mathbf{B}_{i j} \mathbf{V}_{i} \mathbf{V}_{j} \cos \left(\delta_{i}-\delta_{j}\right), \quad \mathbf{i}, \mathbf{j} \in \mathbf{N}_{\mathbf{g}}+1, \ldots, \mathbf{N} .
\end{aligned}
$$

3) Load Equations: Here, we introduce the polynomial function of the static ZIP load model at load bus $i$. A static 
ZIP model is a composite load which consists of constant impedance, constant current and constant power loads, all in parallel. Thus, the load model is nonlinear and the power balance equations for the system loads can be written as

$$
\begin{gathered}
\mathbf{P}_{i}=-\mathbf{P}_{i}\left(\mathbf{V}_{i}\right)=-\mathbf{P}_{i}^{0}\left(\mathbf{a}+\mathbf{b} \mathbf{V}_{i}+c \mathbf{V}_{i}^{2}\right) \\
\mathbf{Q}_{i}=-\mathbf{Q}_{i}\left(\mathbf{V}_{i}\right)=-\mathbf{Q}_{i}^{0}\left(\mathbf{d}+\mathbf{e} \mathbf{V}_{i}+\mathbf{f} \mathbf{V}_{i}^{2}\right) .
\end{gathered}
$$

Here $a, b, c, d, e$, and $f$ are coefficients that express the proportion of various loads combined together. $V_{i}$ is the voltage magnitude of load bud $i$. Equations (1)-(6) constitute the system model, which is a set of DAE's of the form

$$
\begin{gathered}
\dot{\delta}_{i}=\omega_{i}, \quad \mathbf{i} \in 1,2, \ldots, \mathbf{N}_{\mathbf{g}} \\
\dot{\omega}_{i}=\frac{1}{m_{i}}\left(\mathbf{P}_{\mathrm{mi}}-\mathbf{d}_{i} \omega_{i}-\mathbf{P}_{i}\right), \quad \mathbf{i} \in 1,2, \ldots, \mathbf{N}_{\mathbf{g}} \\
-\mathbf{P}_{i}\left(\mathbf{V}_{i}\right)=\sum_{j} \mathbf{G}_{i j} \mathbf{V}_{i} \mathbf{V}_{j} \cos \left(\delta_{i}-\delta_{j}\right) \\
+\sum_{j} \mathbf{B}_{i j} \mathbf{V}_{i} \mathbf{V}_{j} \sin \left(\delta_{i}-\delta_{j}\right), \\
\mathbf{i}, \mathbf{j} \in \mathbf{N}_{\mathbf{g}}+1, \ldots, \mathbf{N} \\
-\mathbf{Q}_{i}\left(\mathbf{V}_{i}\right)=\sum_{j} \mathbf{G}_{i j} \mathbf{V}_{i} \mathbf{V}_{j} \sin \left(\delta_{i}-\delta_{j}\right) \\
-\sum_{j} \mathbf{B}_{i j} \mathbf{V}_{i} \mathbf{V}_{j} \cos \left(\delta_{i}-\delta_{j}\right), \\
\mathbf{i}, \mathbf{j} \in \mathbf{N}_{\mathbf{g}}+1, \ldots, \mathbf{N} .
\end{gathered}
$$

Define the following vectors and matrices:

$$
\begin{aligned}
\delta: & \left(\delta_{1}, \ldots, \delta_{N g}\right)^{T} \\
\theta: & \left(\delta_{N g+1}, \ldots, \delta_{N}\right)^{T} \\
\omega: & \left(\omega_{1}, \ldots, \omega_{N g}\right)^{T} \\
V:= & \left(\mathbf{V}_{N g+1}, \ldots, \mathbf{V}_{N}\right)^{T} \\
\mathbf{P}_{\mathbf{m}}: & =\left(P_{m 1}, \ldots, P_{m N g}\right)^{T} \\
\mathbf{P}^{0}:= & \left(\mathbf{P}_{N g+1}^{o}, \ldots, \mathbf{P}_{N}^{o}\right)^{T} \\
\mathbf{Q}^{o}:= & \left(\mathbf{Q}_{N g+1}^{o}, \ldots, \mathbf{Q}_{N}^{o}\right)^{T} \\
\mathbf{f}(\delta, \theta, \mathbf{V}):= & \left(\mathbf{f}_{1}(\delta, \theta, \mathbf{V}), \ldots, \mathbf{f}_{N g}(\delta, \theta, \mathbf{V})\right)^{T} \\
\mathbf{g}(\delta, \theta, \mathbf{V}):= & \left(\mathbf{f}_{N g+1}(\delta, \theta, \mathbf{V}), \ldots, \mathbf{f}_{N}(\delta, \theta, \mathbf{V})\right)^{T} \\
\mathbf{h}(\delta, \theta, \mathbf{V}):= & \left(\mathbf{h}_{N g+1}(\delta, \theta, \mathbf{V}), \ldots, \mathbf{h}_{N}(\delta, \theta, \mathbf{V})\right)^{T} \\
D:= & \operatorname{diag}\left\{\mathbf{d}_{1}, \ldots, \mathbf{d}_{N g}\right\} \\
M:= & \operatorname{diag}\left\{\mathbf{m}_{1}, \ldots, \mathbf{m}_{N g}\right\} \\
\mathbf{f}_{i}(\delta, \theta, \mathbf{V}):= & \sum_{j} \mathbf{G}_{i j} \mathbf{V}_{i} \mathbf{V}_{j} \cos \left(\delta_{i}-\delta_{j}\right) \\
& +\sum_{j} \mathbf{B}_{i j} \mathbf{V}_{i} \mathbf{V}_{j} \sin \left(\delta_{i}-\delta_{j}\right)+\mathbf{P}_{i}\left(\mathbf{V}_{i}\right) \\
\mathbf{h}_{i}(\delta, \theta, \mathbf{V}):= & \sum_{j} \mathbf{G}_{i j} \mathbf{V}_{i} \mathbf{V}_{j} \sin \left(\delta_{i}-\delta_{j}\right) \\
& -\sum_{j} \mathbf{B}_{i j} \mathbf{V}_{i} \mathbf{V}_{j} \cos \left(\delta_{i}-\delta_{j}\right)+\mathbf{Q}_{i}\left(\mathbf{V}_{i}\right) .
\end{aligned}
$$

We can rewrite (7)-(10) in more compact vector forms

$$
\begin{aligned}
\dot{\delta} & =\omega \\
\dot{\omega} & =\mathbf{M}^{-1}\left(\mathbf{P}_{m}-\mathbf{D} \omega-\mathrm{f}(\delta, \theta, \mathbf{V})\right) \\
0 & =\mathbf{P}^{0}+\mathbf{g}(\delta, \theta, \mathbf{V})
\end{aligned}
$$

$$
0=\mathbf{Q}^{0}+\mathbf{h}(\delta, \theta, \mathbf{V}) .
$$

Remark: Define the Jacobin, $J_{L}$, matrix as follows:

$$
\mathbf{J}_{\mathbf{L}}=\left[\begin{array}{ll}
\frac{\delta \mathbf{g}}{\delta \theta} & \frac{\delta \mathbf{g}}{\delta \mathbf{V}} \\
\frac{\delta \mathbf{h}}{\delta \theta} & \frac{\delta \mathbf{h}}{\delta \mathbf{V}}
\end{array}\right] .
$$

Then, by the implicit function theorem, if the Jacobin $J_{L}$ is nonsingular, locally there exists a $C^{1}$ mapping, $\phi$, such that $(\theta, \mathbf{V})=\phi(\delta)$ and

$$
\begin{aligned}
& 0=\mathbf{P}^{\circ}+\mathbf{g}(\delta, \phi(\delta)) \\
& 0=\mathbf{Q}^{\circ}+\mathbf{h}(\delta, \phi(\delta)) .
\end{aligned}
$$

Thus, we obtain an explicit solution of $\theta$ and $V$ for load flow equations (13)-(14) in terms of generator angle, $\delta$. Substituting this local solution into (11)-(12) yields equivalent ordinary differential equations locally. Conversely, if the $J_{L}$ is singular, then the system will lose load bus voltage causality. The model will then collapse and voltage behavior can no longer be predicted.

\section{B. Piecewise Constant Current Load Equivalent (Current Load Equivalent)}

While the DAE description is accurate for the adopted transient dynamics model, it becomes complex with increasing computation cost as the size of the studied system becomes large. Since the number of algebraic equations is usually much larger than the number of differential equations in a typical power system, there will be a significant reduction of computing burden if one can obtain a local ordinary differential equation equivalent for the original DAE.

We first present one of two equivalencing techniques which can eliminate the algebraic equations such that only internal generator buses are preserved, while approximately retaining the characteristics of ZIP loads. This is achieved by representing the ZIP loads with piecewise constant current loads. In a region where the loads are constant current sources, they can be moved to the generator buses using techniques from circuit theory. Whenever the generator angles move beyond the boundary of the moving hypercube (which defines the region in generator angle space) centered at previous update trajectory point, we update the load current injection by solving the load flow equations. The piecewise linear approximation of a nonlinear load model has also been suggested in [14], although there are significant differences between the two approaches.

We use the following example to illustrate the above concepts. The sample power system consisting of two generators and several ZIP loads can be reduced to two generators, each of which has an additional current injection at its bus, accounting for the effects of load current injection, as shown in Fig. 2. The two additional current injections are functions of the machine angles one and two. 

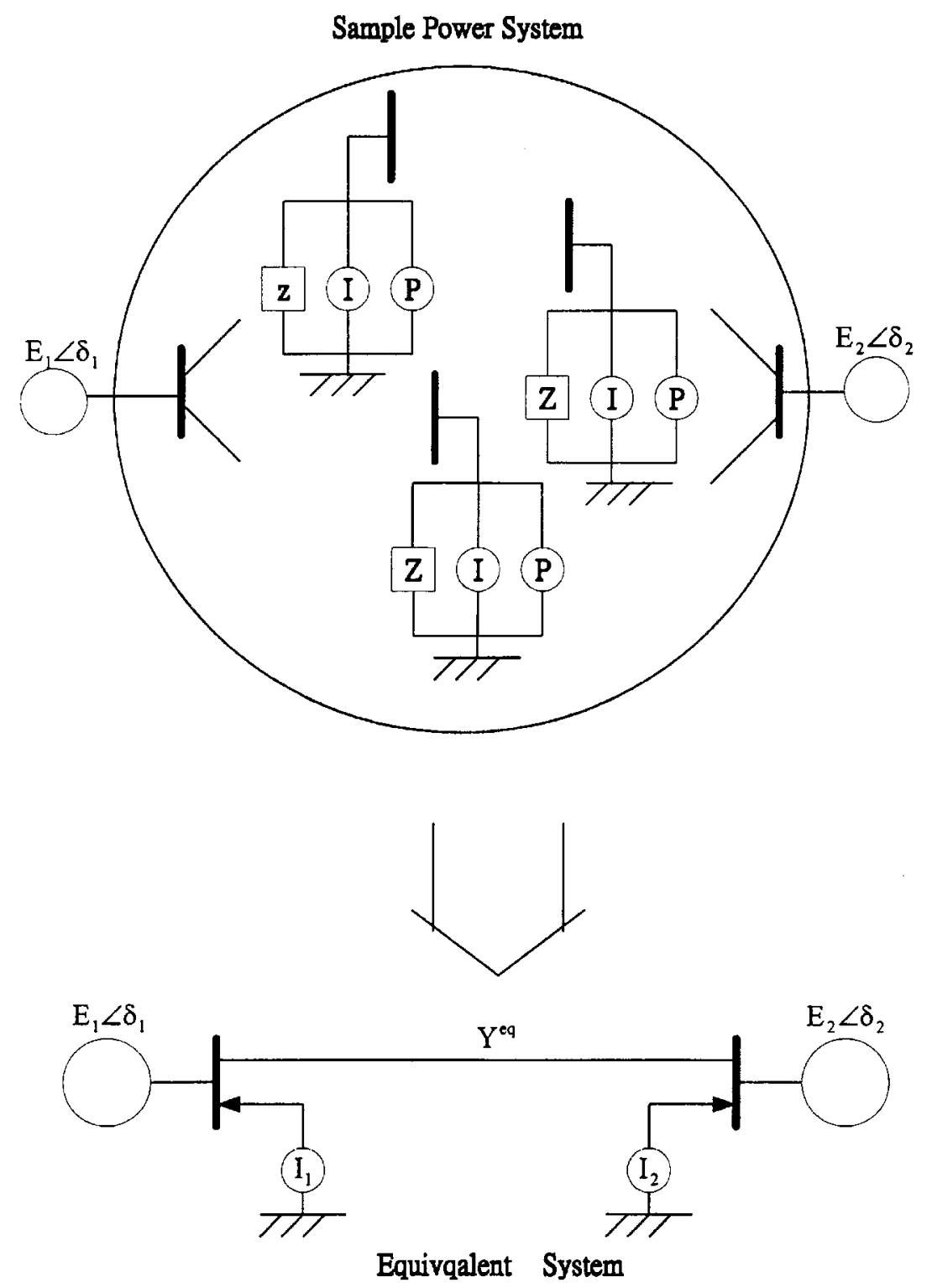

Fig. 2. Sample power system and current load equivalent.

The following are details of the piecewise equivalencing process. For the original system, the bus current phasors are related to the bus voltage phasors and bus admittance matrix $Y$ through

$$
\left[\begin{array}{c}
\mathbf{I}_{g} \\
\mathbf{I}_{l}
\end{array}\right]=\left[\begin{array}{ll}
\mathbf{Y}_{g g} & \mathbf{Y}_{g l} \\
\mathbf{Y}_{l g} & \mathbf{Y}_{l l}
\end{array}\right]\left[\begin{array}{l}
\mathbf{V}_{g} \\
\mathbf{V}_{l}
\end{array}\right]
$$

where the subscript $l$ denotes the load buses to be eliminated and the subscript $g$ denotes the internal generator buses that are to be preserved. After simple algebraic manipulations, the currentvoltage relationship equation (15), reduces to

$$
\mathbf{I}_{g}=\mathbf{Y}^{\mathrm{eq}} \mathbf{V}_{g}+\mathrm{D}^{\mathrm{eq}} \mathbf{I}_{l}
$$

where

$$
\begin{aligned}
& \mathbf{Y}^{\mathrm{eq}}=\mathbf{Y}_{g g}-\mathbf{Y}_{g l} \mathbf{Y}_{l l}^{-1} \mathbf{Y}_{l g} \\
& \mathbf{D}^{\mathrm{eq}}=\mathbf{Y}_{g l} \mathbf{Y}_{l l}^{-1} .
\end{aligned}
$$

Equation (16) has two terms, with the first term representing the contributions to generator current injections from generator voltages and the second term representing the load current's contributions. By using (16), the swing equations for the $i$ th generator can be readily derived as

$$
\dot{\delta}_{i}=\omega_{i}
$$

$$
\dot{\omega}_{i}=\frac{1}{\mathbf{m}_{i}}\left(\mathbf{P}_{\mathrm{mi}}-\mathbf{d}_{i} \omega_{i}-\mathbf{P}_{i}\right)
$$

where

$$
\begin{aligned}
\mathbf{P}_{i}= & \operatorname{Real}\left(\mathbf{V}_{g i} I_{g i}^{*}\right) \\
= & \left|\mathbf{V}_{g i}\right| \sum_{j=1}^{N g}\left|\mathbf{Y}_{i j}^{\mathrm{eq}}\right|\left|\mathbf{V}_{g j}\right| \cos \left(\delta_{i}-\delta_{j}-\phi_{i j}^{\mathrm{eq}}\right) \\
& +\left|\mathbf{V}_{g i}\right| \sum_{j=N g+1}^{N}\left|\mathbf{D}_{i j}^{\mathrm{eq}}\right|\left|\mathbf{I}_{l j}\right|(\delta) \cos \left(\delta_{i}-\varphi_{i j}(\delta)-\psi_{i j}^{\mathrm{eq}}\right)
\end{aligned}
$$


and $\left|\mathbf{Y}_{i j}^{\mathrm{eq}}\right| \angle \phi_{i j}^{\mathrm{eq}}$ are the entries of matrix $\mathbf{Y}^{\mathrm{eq}}$ and $\left|\mathbf{D}_{i j}^{\mathrm{eq}}\right| \angle \psi_{i j}^{\mathrm{eq}}$ are the entries of matrix $\mathrm{D}^{\mathrm{eq}}$. Note that the load current $\left|\mathbf{I}_{l j}\right|<\varphi_{l j}$ is, in fact, a function of generator angles due to the ZIP loads. The principle of the technique is that we approximate the load current, which is a function of generator angles, by a constant value whenever the generator angles lie inside the moving hypercube along the projected trajectory on generator angle space.

The hypercube is a set of generator angles defined in angle space as

$$
\left\{\delta_{i}:\left|\delta_{i}-\delta_{i}^{\circ}\right| \leq \gamma\right\}
$$

where the $\gamma$ is an update parameter with unit radian or degree specified by monitors, and $\delta_{i}^{o}$ is the angle of last update trajectory point. Fig. 3 illustrates the moving hypercube along the trajectory. With the approximation of constant current load in the moving hypercube and update of currents whenever exceeding the hypercube, the transient dynamics are then described by piecewise swing (19)-(20). When the generator angles move beyond the boundary of the current hypercube, the constant load current will be recomputed by the following procedures. First, we choose the current generator angles as the new center of the moving hypercube and then solve load flow equations (13)-(14) using current generator voltage magnitudes and angles as knowns for load bus voltage magnitudes and angles at a given load bus. Then, the new constant load at the $j$ th bus is computed as

$$
\left|\mathbf{I}_{i j}\right| \angle \varphi_{i j}=\frac{\sqrt{\mathbf{P}_{j}^{2}\left(\mathbf{V}_{j}\right)+\mathbf{Q}_{j}^{2}\left(\mathbf{V}_{j}\right)}}{\mathbf{V}_{i j}} \angle\left(\theta_{l j}-\phi_{l j}\right)
$$

where $\phi_{l j}$ is the power factor angle at bus $j$ given by

$$
\phi_{l j}=\tan ^{-1}\left(\frac{\mathbf{Q}_{j}\left(\mathbf{V}_{j}\right)}{\mathbf{P}_{j}\left(\mathbf{V}_{j}\right)}\right) .
$$

This completes the equivalencing process.

\section{Piecewise Constant Transfer Admittance Equivalent (Admittance Equivalent)}

In this subsection, we present the other equivalencing technique which can eliminate algebraic equations so that only the differential equations describing the dynamics of internal generator angles are preserved, while approximately retaining the nonlinear characteristics of ZIP loads. This is achieved by absorbing the ZIP loads into piecewise constant transfer admittance. Fig. 4 shows that sample power system consisting of two generators and several ZIP loads. This power system can be reduced to two generators, each of which is connected by transmission line with equivalent admittance and incremental equivalent admittance accounting for the nonlinear characteristics of the ZIP load.

The equivalencing technique is described as follows. First, from circuit theory, one obtains

$$
\left[\begin{array}{c}
\mathbf{I}_{g} \\
\mathbf{I}_{l}
\end{array}\right]=\left[\begin{array}{ll}
\mathbf{Y}_{g g} & \mathbf{Y}_{g l} \\
\mathbf{Y}_{l g} & \mathbf{Y}_{l l}
\end{array}\right]\left[\begin{array}{l}
\mathbf{V}_{g} \\
\mathbf{V}_{l}
\end{array}\right]
$$

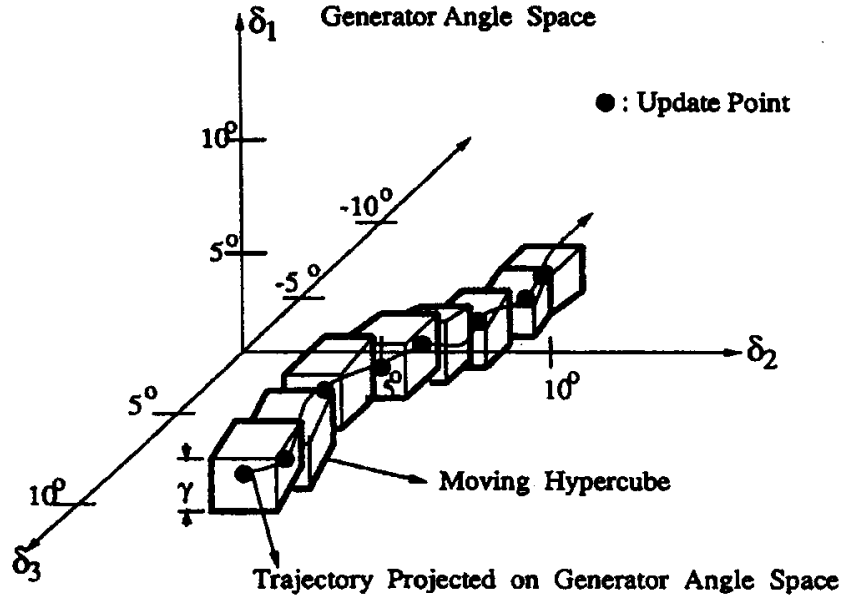

Fig. 3. Moving hypercubes in the current load equivalent technique.

After algebraic manipulations, (21) yields

$$
\mathbf{I}_{g}=\mathbf{Y}^{\mathrm{eq}}\left(\mathbf{V}_{l}\right) \mathbf{V}_{g}
$$

where $Y^{\mathrm{eq}}$ is a function of voltage magnitude at load buses and is derived as

$$
\mathbf{Y}^{\mathrm{eq}}\left(\mathbf{V}_{l}\right)=\mathbf{Y}_{g g}-\mathbf{Y}_{g l} \mathbf{Y}\left(\mathbf{V}_{l}\right)_{l l}^{-1} \mathbf{Y}_{l g}
$$

where

$$
\mathbf{Y}\left(\mathbf{V}_{l}\right)_{l l}^{-1}=\left[\operatorname{diag}\left[\frac{\mathbf{P}_{l}\left(\mathbf{V}_{l}\right)-\mathbf{j} \mathbf{Q}_{l}\left(\mathbf{V}_{l}\right)}{\mathbf{V}_{l}}\right]-\mathbf{Y}_{l l}\right]^{-1}
$$

When the generator angles move beyond the boundary of the hypercube centered at the previous update trajectory point, the equivalent admittance $Y^{\text {eq }}$ is updated by first-order approximation to avoid matrix inversion. Assume that $V_{l}^{o}$ is prefault load voltage magnitude and $\Delta V_{l}$ is incremental load voltage magnitude due to angle swing, which can be obtained by solving load flow. A first-order Taylor expansion for $\mathbf{Y}^{\mathrm{eq}}\left(\mathbf{V}_{l}^{o}+\Delta \mathbf{V}_{l}\right)$ is obtained as

$$
\mathbf{Y}^{\mathrm{eq}}\left(\mathbf{V}_{l}^{o}+\Delta \mathbf{V}_{l}\right)=\mathbf{Y}_{o}^{\mathrm{eq}}+\Delta \mathbf{Y}^{\mathrm{eq}}
$$

where

$$
\begin{aligned}
\mathbf{Y}_{o}^{\mathrm{eq}} & =\mathbf{Y}_{g g}-\mathbf{Y}_{g l}\left(\mathbf{Y}_{l l}-\mathbf{Y}_{l}^{o}\right)^{-1} \mathbf{Y}_{l g} \\
\Delta \mathbf{Y}^{\mathrm{eq}} & =\mathbf{Y}_{g l}\left(\mathbf{Y}_{l l}-\mathbf{Y}_{l}^{o}\right)^{-1} \mathbf{S} \operatorname{diag}\left[\delta \mathbf{V}_{l i}\right]\left(\mathbf{Y}_{l l}-\mathbf{Y}_{l}^{o}\right)^{-1} \mathbf{Y}_{e g}
\end{aligned}
$$

and

$$
\begin{gathered}
\mathbf{Y}_{l}^{o}=\operatorname{diag}\left[\frac{\mathbf{P}_{l}\left(\mathbf{V}_{l}\right)-\mathbf{j} \mathbf{Q}_{l}\left(\mathbf{V}_{l}\right)}{\mathbf{V}_{l}^{2}}\right] \\
\mathbf{S}=\operatorname{diag}\left[\frac{\partial\left(\frac{\mathbf{P}_{l}\left(\mathbf{V}_{l}\right)-\mathbf{j} \mathbf{Q}_{l}\left(\mathbf{V}_{l}\right)}{\mathbf{V}_{l}^{2}}\right)}{\partial\left(\mathbf{V}_{l}\right)^{2}}\right] .
\end{gathered}
$$

By using (25), the swing equations for the $i$ th generator can be readily derived as

$$
\dot{\delta}_{i}=\omega_{i}
$$

$$
\mathbf{m}_{\mathbf{i}} \dot{\omega}_{i}+\mathbf{d}_{i} \omega_{i}=\mathbf{P}_{\mathrm{mi}}-\mathbf{P}_{i}
$$

where

$$
\mathbf{P}_{i}=\mathbf{P}_{o i}+\Delta \mathbf{P}_{i}
$$



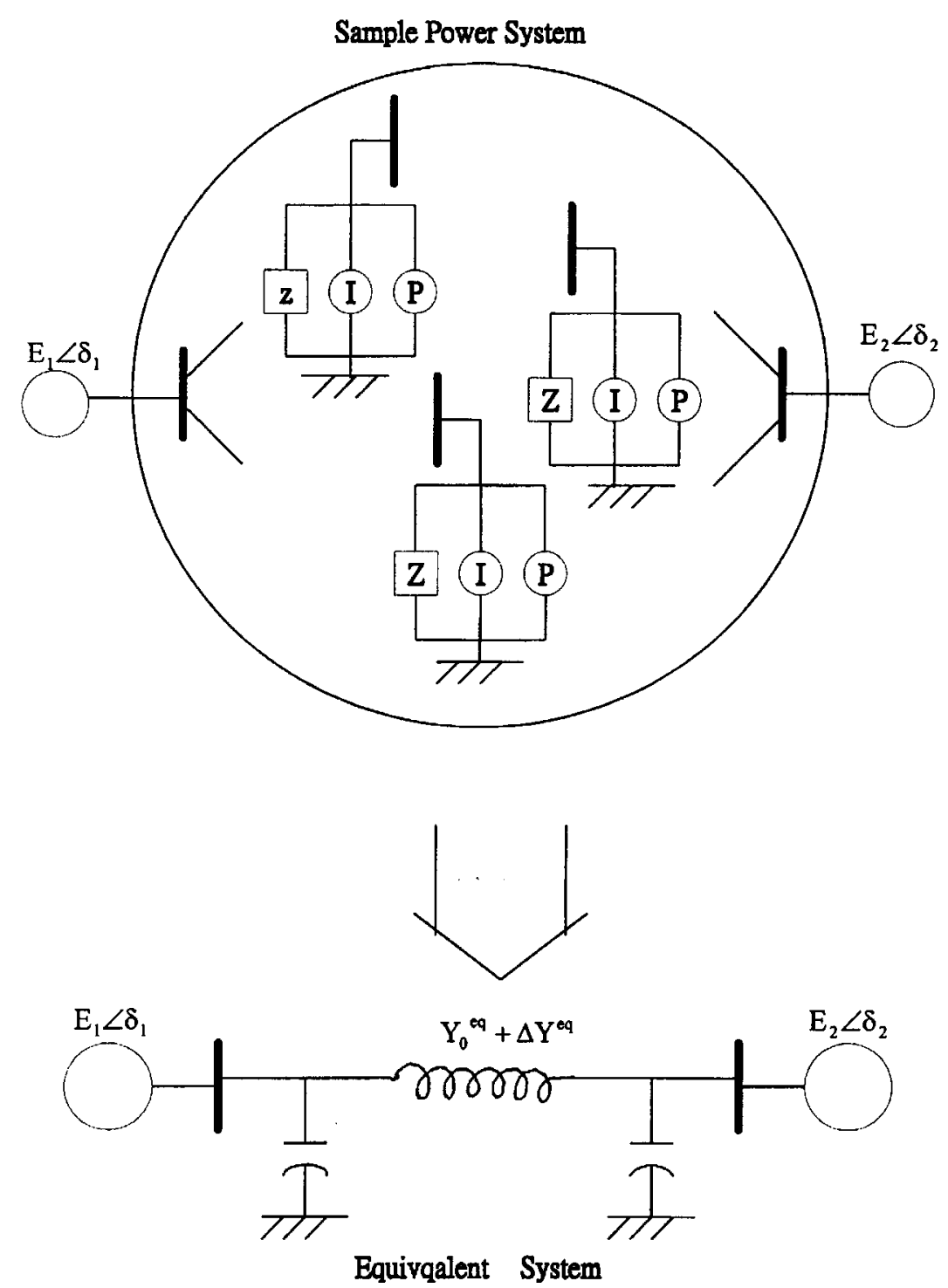

Fig. 4. Sample power system and admittance equivalent technique.

and

$$
\begin{aligned}
\mathbf{P}_{o i}= & \sum_{j=1}^{N g-1} \mathbf{G}_{o i j}^{\mathrm{eq}} \mathbf{V}_{i} \mathbf{V}_{j} \cos \left(\delta_{i}-\delta_{j}\right) \\
& +\sum_{j=1}^{N g-1} \mathbf{B}_{o i j}^{\mathrm{eq}} \mathbf{V}_{i} \mathbf{V}_{j} \sin \left(\delta_{i}-\delta_{j}\right) \\
\Delta \mathbf{P}_{i}= & \sum_{j=1}^{N g-1} \Delta \mathbf{G}_{o i j}^{\mathrm{eq}} \mathbf{V}_{i} \mathbf{V}_{j} \cos \left(\delta_{i}-\delta_{j}\right) \\
& +\sum_{j=1}^{N g-1} \Delta \mathbf{B}_{o i j}^{\mathrm{eq}} \mathbf{V}_{i} \mathbf{V}_{j} \sin \left(\delta_{i}-\delta_{j}\right)
\end{aligned}
$$

where $\mathbf{G}_{o i j}^{\mathrm{eq}}+\mathbf{j} \mathbf{B}_{o i j}^{\mathrm{eq}}=\mathbf{Y}_{o i j}^{\mathrm{eq}}$ are the entries of $\mathbf{Y}_{o}^{\mathrm{eq}}$ and $\Delta \mathbf{G}_{o i j}^{\mathrm{eq}}+$ $\mathbf{j} \Delta \mathbf{B}_{o i j}^{\mathrm{eq}}=\Delta \mathbf{Y}_{o i j}^{\mathrm{eq}}$ are the entries of $\Delta \mathbf{Y}^{\mathrm{eq}}$.

With the approximation of equivalent admittance in the hypercube and update of equivalent admittance whenever the hypercube is exceeded, the transient dynamics are then described by piecewise swing (26)-(27). This completes the equivalencing process.

\section{Criteria for Transient Stability Prediction}

This section establishes the criteria for stability identification of a developing swing. Traditionally, transient stability is concerned with the capability of generators to capture synchronism following a large disturbance, such as three-phase short circuit, loss of generator, or sudden change of load. In this case, the criteria can be so defined that a swing after a fault is determined as an unstable swing if the relative angles of different generator groups separate indefinitely with times. Otherwise, it is identified as a stable swing. However, in practice, a postfault swing which does not cause a synchronism problem may exhibit pole slipping, voltage dip, and transmission line overloading. These conditions may initiate important relay operations such as out-of-step blocking or tripping, thus causing undesirable situations. Therefore, a more stringent stability criterion is needed to meet real-time operation requirements and we define the practical transient stability criterion in terms of the security constraint region in state-space for prediction use.

1) Generator Angle Security Constraint: Considerations of out-of-synchronism and pole-slipping conditions impose limits 
on the machine angles of internal buses with respect to neighboring buses as

$$
\left|\delta_{k}\right| \leq \pi
$$

Denote the security region inside the limits by $R_{\delta}$, i.e.,

$$
\mathbf{R}_{\delta}:=\left\{\delta:\left|\delta_{k}\right| \leq \pi\right\} .
$$

2) Generator Frequency Deviation Constraint: The frequency deviation that accompanies system disturbances is caused by the imbalance between load and generation. Excess generation will cause a rising frequency. Excess load, however, will cause the frequency to drop. Both cases can cause permanent damage to steam turbines. Therefore, limits are imposed on the generator frequency deviation $\omega_{k}$ as

$$
\psi \leq\left|\omega_{k}\right| \leq \alpha, \quad \mathbf{k}=1,2, \ldots, \mathbf{N}_{g} .
$$

Denote the region inside the limits by $R_{\varpi}$, i.e.,

$$
\mathbf{R}_{\omega}:=\left\{\omega: \psi \leq \omega_{k} \leq \alpha\right\}, \quad \mathbf{k}=1,2, \ldots, \mathbf{N}_{g} .
$$

3) Load Bus Voltage Magnitude Security Constraint: Consideration of operating and voltage dip impose the limits on load bus voltage magnitude $V_{k}$ as

$$
1-\Delta \leq \mathbf{V}_{k} \leq 1+\varepsilon, \quad \mathbf{k}=\mathbf{N}_{g+1}, \ldots, \mathbf{N} .
$$

Denote the corresponding security region in space $V$ by $\mathbf{R v}$, i.e.,

$$
\mathbf{R v}:=\left\{V: 1-\Delta \leq \mathbf{V}_{k} \leq 1+\varepsilon, \mathbf{k}=\mathbf{N}_{g+1}, \ldots, \mathbf{N}\right\} .
$$

4) Load Bus Voltage Angle Security Constraint: Thermal considerations limit the amount of current flowing through transmission lines and transformers. By calculations and approximations, the line flow constraints may be expressed approximately in terms of phase angle differences, implying that there are limits imposed on load bus voltage angles $\theta_{k}$ as

$$
\left|\theta_{k}-\theta \mathbf{j}\right| \leq \beta, \quad \mathbf{k}, \mathbf{j}=\mathbf{N}_{g+1}, \ldots, \mathbf{N}
$$

Denote the security region in $\theta$ space by $R_{\theta}$, i.e.

$$
\mathbf{R}_{\theta}:=\left\{\theta:\left|\theta_{k}-\theta_{j}\right| \leq \beta, \mathbf{k}, \mathbf{j}=\mathbf{N}_{g+1}, \ldots, \mathbf{N}\right\} .
$$

5) Practical Transient Stability Criterion: We define the security set in $(\delta, \omega, \theta, \mathbf{V})$ space as

$$
\mathbf{R}_{\mathrm{TS}}:=\mathbf{R}_{\delta} \times \mathbf{R}_{\omega} \times \mathbf{R}_{\theta} \times \mathbf{R}_{V} .
$$

With the preceding concepts, we precisely formulate the real-time transient stability prediction problem in the following way.

Problem: Given a set of real-time measurement vectors $\quad \delta(\mathbf{k} \Delta \mathbf{T}), \omega(\mathbf{k} \Delta \mathbf{T}), \theta(\mathbf{k} \Delta \mathbf{T}), \mathbf{V}(\mathbf{k} \Delta \mathbf{T}) \quad$ where $k=1,2 \ldots, M, \Delta T$ is the sampling period and $M \Delta T$ is the length of the observation window after a fault. For $k=0$, the measurement vector comprises the prefault steady state. For $k=1,2, \ldots, M$, the measurement vectors comprise the postfault observed dynamic states. Usually, stability prediction is based on an observed eight-cycle window of real-time measurements. Three consecutive measurements, four cycles apart, are taken, that is, $M=3$. The objective is to find a methodology that can process the observed real-time measurements to calcu- late the time history of $\delta(\mathbf{k} \Delta \mathbf{T}), \omega(\mathbf{k} \Delta \mathbf{T}), \theta(\mathbf{k} \Delta \mathbf{T}), \mathbf{V}(\mathbf{k} \Delta \mathbf{T})$, $k=M+1, M+2, \ldots, M+N$ over a prediction window with length $N \Delta T$ before the transient swing actually occurs, so that one can predict whether a developing swing is stable or not by the following criteria.

Stable swing: $\delta(\mathbf{k} \Delta \mathbf{T}), \omega(\mathbf{k} \Delta \mathbf{T}), \theta(\mathbf{k} \Delta \mathbf{T}), \mathbf{V}(\mathbf{k} \Delta \mathbf{T}) \quad \epsilon$ $\mathbf{R}_{\mathrm{TS}}$, for $k=M+1, M+2, \ldots, M+N$, otherwise, the swing is determined to be unstable.

Remark: In practice, since there are uncertainties in the model and noise in the real-time measurements, it is safer to predict the stability of a swing by processing consecutive observed measurements as different initial conditions and going through the methodology in turn. When these are complete, the outcome of stability prediction is checked. If the number of stability status is greater than the instability status, the swing is identified as being stable. Otherwise, it is determined to be unstable.

In the following two sections, we propose three methodologies, decoupled integration, current load equivalent, and admittance equivalent for solving the above stability prediction problem.

\section{IMPLICITLY DeCOUPLED PQ INTEGRATION (DECOUPLED INTEGRATION) TECHNIQUE}

Numerical schemes for simultaneous solution of DAE's are currently available [15]. The simplest one, using the implicit trapezoidal rule, has proven to be numerically stable, as well as having only minor errors for solving DAE's, such as the wellknown Dommel's technique [16]. This scheme involves forming an update matrix and performing matrix factorization, such as LU decomposition, at several time steps. Hence, the implicit integration methods are often considered to be computationally more expensive than explicit integration methods such as the Runge-Kutta method. Using the proposed decoupled integration method, it is demonstrated both theoretically and numerically that, under certain conditions, a constant and decoupled form of the update matrix can be used to speed up the implicit integration technique. Specifically, the algorithm is shown to be convergent under some reasonable assumptions.

\section{A. Decoupled Integration Algorithm}

The decoupled integration technique is outlined below.

First we define the following functions for the ease of statements in the algorithm

$$
\Delta_{n}(\delta, \omega, \theta, \mathbf{V}, \mathbf{s})=\left(\delta-\delta_{n}\right)-\frac{\mathbf{s}}{2}\left(\omega+\omega_{n}\right)
$$

$$
\begin{aligned}
\Omega_{n}(\delta, \omega, \theta, \mathbf{V}, \mathbf{s})= & \left(\omega-\omega_{n}\right)-\frac{\mathbf{S}}{2} \mathbf{M}^{-1}\left[2 \mathbf{P}_{m}-\mathbf{D}\left(\omega-\omega_{n}\right)\right. \\
& \left.-\mathbf{f}(\delta, \theta, \mathbf{V})-\mathbf{f}\left(\delta_{n}, \theta_{n}, \mathbf{V}_{n}\right)\right]
\end{aligned}
$$

$$
\mathbf{G}_{n}(\delta, \omega, \theta, \mathbf{V}, \mathbf{s})=\mathbf{P}^{o}+\mathbf{g}(\delta, \theta, \mathbf{V})
$$

$$
\mathbf{H}_{n}(\delta, \omega, \theta, \mathbf{V}, \mathbf{s})=\mathbf{Q}^{\circ}+\mathbf{h}(\delta, \theta, \mathbf{V}) .
$$


Step 1) Discretize (11)-(14) by the implicit trapezoidal rule, yielding

$$
\begin{aligned}
\frac{1}{\mathbf{s}}\left(\delta_{n}-\delta_{n-1}\right)= & \frac{1}{2}\left(\omega_{n}+\omega_{n-1}\right) \\
\frac{1}{\mathbf{s}}\left(\omega_{n}-\omega_{n-1}\right)= & \frac{1}{2} \mathbf{M}^{-1}\left[2 \mathbf{P}_{m}-\mathbf{D}\left(\omega_{n}+\omega_{n-1}\right)\right. \\
& \left.-\mathbf{f}\left(\delta_{n}, \theta_{n}, \mathbf{V}_{n}\right)-\mathbf{f}\left(\delta_{n-1}, \theta_{n-1}, \mathbf{V}_{n-1}\right)\right]
\end{aligned}
$$

$$
\begin{aligned}
& 0=\mathbf{P}^{\circ}+\mathbf{g}\left(\delta_{n}, \theta_{n}, \mathbf{V}_{n}\right) \\
& 0=\mathbf{Q}^{\circ}+\mathbf{h}\left(\delta_{n}, \theta_{n}, \mathbf{V}_{n}\right)
\end{aligned}
$$

where $s$ is the step size and the subscripts $n-1$ and $n$ denote the time index of two successive trajectory points. The postfault trajectory in state space is an ordered set of trajectory points, i.e.,

$$
\left\{\left(\delta_{n}, \omega_{n}, \theta_{n}, \mathbf{V}_{n}\right), \mathbf{n}=1,2 \ldots\right\}
$$

which satisfies (32)-(35) recursively. Partition functions (28)-(31) into two groups. Group A consisting of functions (28)-(30) and Group B consisting of function (31).

Step 2) Evaluate the Jacobin matrix $J_{A}^{o}$ of Group A with respect to $(\delta, \omega, \theta)$ at the prefault operating point, $\left(\delta_{o}, \omega_{o}, \theta_{0}, V_{o}\right)$. Note that we do not need to assume knowledge of the fault location

$$
\mathbf{J}_{A}^{\circ}=\left[\begin{array}{ccc}
\mathbf{I} & \frac{-\mathbf{S}}{\mathbf{I}} & \mathbf{O} \\
\frac{\mathbf{s}}{2} \mathbf{M}^{-1} \frac{\partial \mathbf{f}}{\partial \delta} & \mathbf{I}+\frac{\mathbf{s}}{2} \mathbf{M}^{-1} \mathbf{D} & \frac{\mathbf{s}}{2} \mathbf{M}^{-1} \frac{\partial \mathbf{f}}{\partial \theta} \\
\frac{\partial \mathbf{g}}{\partial \delta} & \mathbf{O} & \frac{\partial \mathbf{g}}{\partial \theta}
\end{array}\right]
$$

and also the Jacobian matrix $J_{B}^{\circ}$ of Group B with respect to $V$

$$
\mathbf{J}_{B}^{o}=\frac{\partial \mathbf{h}}{\partial \mathbf{V}} .
$$

Step 3) Find the next trajectory point $\left(\delta_{n+1}, \omega_{n+1}, \theta_{n+1}, \mathbf{V}_{n+1}\right) \quad$ by quasi-Newton method using $\left(\delta_{n}, \omega_{n}, \theta_{n}, V_{n}\right)$ as the initial point. Setting

$$
\left(\delta_{n}^{o}, \omega_{n}^{o}, \theta_{n}^{o}, \mathbf{V}_{n}^{o}\right)=\left(\delta_{n}, \omega_{n}, \theta_{n}, \mathbf{V}_{n}\right)
$$

the quasi-Newton iterations are

$$
\begin{aligned}
{\left[\begin{array}{l}
\delta_{n}^{k+1} \\
\omega_{n}^{k+1} \\
\theta_{n}^{k+1}
\end{array}\right] } & =\left[\begin{array}{c}
\delta_{n}^{k} \\
\omega_{n}^{k} \\
\theta_{n}^{k}
\end{array}\right]-\left(\mathbf{J}_{A}^{o}\right)^{-1}\left[\begin{array}{l}
\Delta_{n}\left(\delta_{n}^{k}, \omega_{n}^{k}, \theta_{n}^{k}, \mathbf{V}_{n}^{k}\right) \\
\Omega_{n}\left(\delta_{n}^{k}, \omega_{n}^{k}, \theta_{n}^{k}, \mathbf{V}_{n}^{k}\right) \\
\mathbf{G}_{n}\left(\delta_{n}^{k}, \omega_{n}^{k}, \theta_{n}^{k}, \mathbf{V}_{n}^{k}\right)
\end{array}\right] \\
\mathbf{V}_{n}^{k+1} & =\mathbf{V}_{n}^{k}-\left(\mathbf{J}_{B}^{o}\right)^{-1}\left[\mathbf{H}_{n}\left(\delta_{n}^{k}, \omega_{n}^{k}, \theta_{n}^{k}, \mathbf{V}_{n}^{k}\right)\right]
\end{aligned}
$$$$
k=0,1,2, \ldots \text { until the convergence condition is }
$$
satisfied.

Step 4) Perform Step 3) to compute successive trajectory points $\left(\delta_{n}, \omega_{n}, \theta_{n}, V_{n}\right)$ until the prediction interval is finished, or until convergence does not occur for some value of $n$. If convergence fails to occur, e.g., for $n=M+1$, then divide the step size by 2 and repeat Steps 2) and 3), using $\left(\delta_{m}, \omega_{m}, \theta_{m}, V_{m}\right)$ as the initial condition. If convergence still does not occur, then check the singularity of $J_{L}$ at $\left(\delta_{m}, \omega_{m}, \theta_{m}, V_{m}\right)$. If $J_{L}$ is nearly singular, then stop the algorithm and give the prediction of voltage instability. If $J_{L}$ is nonsingular, reduce the step size until convergence occurs in Step 3). Repeat Step 4) until the prediction interval is finished or voltage instability is determined.

Remark 3.1: The advantages of this technique are the numerical stability of implicit integration and a large reduction of computational burden because there is no new function evaluation during Steps 3) and 4).

Remark 3.2: In our numerical experiments, we required only one evaluation for $J_{A}^{\circ} \& J_{B}^{o}$ evaluated at the prefault condition in order to predict several seconds into the future. This implies that we can compute various $J_{A}^{o} \& J_{B}^{o}$ and store them in the prefault stage for different configurations, so that computing time can be saved in the postfault prediction stage.

Before demonstrating the effectiveness of the above algorithm by numerical experiments, we would like to theoretically show the feasibility of the algorithm by the following lemmas and theorems.

Define the system Jacobin $J_{s}$ of (28)-(31) with respect to $(\delta, \omega, \theta, V)$ as follows:

$$
\mathbf{J}_{s}=\left[\begin{array}{cccc}
\mathbf{I} & -\frac{\mathbf{s}}{2} \mathbf{I} & 0 & 0 \\
\frac{\mathbf{s}}{2} \mathbf{M}^{-1} \frac{\partial \mathbf{f}}{\partial \delta} & \mathbf{I}+\frac{\mathbf{s}}{2} \mathbf{M}^{-1} \mathbf{D} & \frac{\mathbf{s}}{2} \mathbf{M}^{-1} \frac{\partial \mathbf{f}}{\partial \theta} & \frac{\mathbf{s}}{2} \mathbf{M}^{-1} \frac{\partial \mathbf{f}}{\partial \mathbf{v}} \\
\frac{\partial \mathbf{g}}{\partial \delta} & 0 & \frac{\partial \mathbf{g}}{\partial \theta} & \frac{\partial \mathbf{g}}{\partial \mathbf{V}} \\
\frac{\partial \mathbf{h}}{\partial \delta} & 0 & \frac{\partial \mathbf{h}}{\partial \theta} & \frac{\partial \mathbf{h}}{\partial \mathbf{V}}
\end{array}\right]
$$

Lemma 3.1 (Existence of the Successive Trajectory Point): If $J_{L}$ is evaluated as nonsingular at a trajectory point, $\left(\delta_{n}, \omega_{n}, \theta_{n}, V_{n}\right)$, then there exists an unique successive trajectory point $\left(\delta_{n+1}, \omega_{n+1}, \theta_{n+1}, V_{n+1}\right)$, which can be expressed as function of $s$ for sufficiently small $s$.

Proof: Note that

$$
\begin{aligned}
\Delta_{n}\left(\delta_{n}, \omega_{n}, \theta_{n}, \mathbf{V}_{n}, 0\right) & =0 \\
\Omega_{n}\left(\delta_{n}, \omega_{n}, \theta_{n}, \mathbf{V}_{n}, 0\right) & =0 \\
\mathbf{G}_{n}\left(\delta_{n}, \omega_{n}, \theta_{n}, \mathbf{V}_{n}, 0\right) & =0 \\
\mathbf{H}_{n}\left(\delta_{n}, \omega_{n}, \theta_{n}, \mathbf{V}_{n}, 0\right) & =0
\end{aligned}
$$

and

$$
\left.\mathbf{J}_{s}\right|_{\left(\delta_{n}, \omega_{n}, \theta_{n}, V_{n}, 0\right)}=\left[\begin{array}{cccc}
\mathbf{I} & 0 & 0 & 0 \\
0 & \mathbf{I} & 0 & 0 \\
\frac{\partial \mathbf{g}}{\partial \delta} & 0 & \frac{\partial \mathbf{g}}{\partial \theta} & \frac{\partial \mathbf{g}}{\partial \mathbf{V}} \\
\frac{\partial \mathbf{h}}{\partial \delta} & 0 & \frac{\partial \mathbf{h}}{\partial \theta} & \frac{\partial \mathbf{h}}{\partial \mathbf{V}}
\end{array}\right]
$$

So,

$$
\begin{aligned}
\left.\operatorname{det} \mathbf{J}_{s}\right|_{\left(\delta_{n}, \omega_{n}, \theta_{n}, V_{n}, 0\right)} & =\operatorname{det}\left[\begin{array}{ll}
\mathbf{I} & 0 \\
0 & \mathbf{I}
\end{array}\right] \operatorname{det}\left[\begin{array}{ll}
\frac{\partial \mathbf{g}}{\partial \theta} & \frac{\partial \mathbf{g}}{\partial \mathbf{V}} \\
\frac{\partial \mathbf{h}}{\partial \theta} & \frac{\partial \mathbf{h}}{\partial \mathbf{V}}
\end{array}\right] \\
& =\operatorname{det} \mathbf{J}_{L} \\
& \neq 0 .
\end{aligned}
$$

Hence, the result follows from the implicit function theorem.

Lemma 3.2 (Nonsingularity of $J_{A}^{0} \& J_{B}^{0}$ ): If the following conditions are satisfied: 
i) the angle difference, $\theta_{o i}-\theta_{o j}$ between any two connected buses at prefault stage are small such that $\cos \left(\theta_{o i}-\theta_{o j}\right) \approx$ 1 and $\sin \left(\theta_{o i}-\theta_{o j}\right) \approx 0$

ii) voltage magnitude, $\mathbf{V}_{o i}$ and $\mathbf{V}_{o j}$ are around one per unit. Then there exists a small step size $s$ such that $J_{A}^{0} \& J_{B}^{0}$ are nonsingular.

Proof: First consider the entries of $\partial \mathrm{g} / \partial \theta$ and $\partial \mathbf{h} / \partial \mathbf{V}$ evaluated at the prefault operating point.

For $i \neq j$

$$
\begin{aligned}
\frac{\partial \mathbf{g}_{i}}{\partial \theta_{j}}= & -\mathbf{V}_{o i} \mathbf{V}_{o j} \mathbf{G}_{i j} \sin \left(\theta_{o i}-\theta_{o j}\right) \\
& -\mathbf{V}_{o i} \mathbf{V}_{o j} \mathbf{B}_{i j} \cos \left(\theta_{o i}-\theta_{o j}\right) \\
\approx & -\mathbf{B}_{i j} \\
\frac{\partial \mathbf{h}_{i}}{\partial \mathbf{V}_{j}}= & \mathbf{V}_{o i} \mathbf{G}_{i j} \sin \left(\theta_{o i}-\theta_{o j}\right)-\mathbf{V}_{o i} \mathbf{B}_{i j} \cos \left(\theta_{o i}-\theta_{o j}\right) \\
\approx & -\mathbf{B}_{i j} .
\end{aligned}
$$

For $i=j$

$$
\begin{aligned}
\frac{\partial \mathbf{g}_{i}}{\partial \theta_{j}}= & \sum_{k \neq i} \mathbf{V}_{o i} \mathbf{V}_{o k} \mathbf{G}_{i k} \sin \left(\theta_{o i}-\theta_{o k}\right) \\
& +\sum_{k \neq i} \mathbf{V}_{o i} \mathbf{V}_{o k} \mathbf{B}_{i k} \cos \left(\theta_{o i}-\theta_{o k}\right) \\
\approx & \sum_{k \neq i} \mathbf{B}_{i k} \\
\frac{\partial \mathbf{h}_{i}}{\partial \mathbf{V}_{i}}= & -2 \mathbf{V}_{o i} \mathbf{B}_{i i}+\sum_{k \neq i} \mathbf{V}_{o i} \mathbf{G}_{i k} \sin \left(\theta_{o i}-\theta_{o k}\right) \\
& -\sum_{k \neq i} \mathbf{V}_{o i} \mathbf{B}_{i k} \cos \left(\theta_{o i}-\theta_{o k}\right) \\
\approx & -2 \mathbf{B}_{i i}-\sum_{k \neq i} \mathbf{B}_{i k} .
\end{aligned}
$$

Hence

$$
\begin{aligned}
& \left|\frac{\partial \mathbf{g}_{i}}{\partial \theta_{i}}\right| \approx\left|\sum_{k \neq i} \mathbf{B}_{i k}\right|=\sum_{k \neq i}\left|\mathbf{B}_{i k}\right| \\
& \left.\quad \because \text { all } \mathbf{B}_{i k} \text { have the same signs }\right) \\
& \sum_{j \neq i}\left|\frac{\partial \mathbf{g}_{i} \mid}{\partial \theta_{i}}\right| \approx \sum_{j \neq i}\left|-\mathbf{B}_{i j}\right|=\sum_{j \neq i}\left|\mathbf{B}_{i j}\right| \\
& \left|\frac{\partial \mathbf{h}_{i}}{\partial \mathbf{V}_{i}}\right| \approx\left|-2 \mathbf{B}_{i i}-\sum_{k \neq i} \mathbf{B}_{i k}\right|=\left|2 \mathbf{B}_{i i}\right|+\sum_{k \neq i}\left|\mathbf{B}_{i k}\right| \\
& \quad\left(\because \text { all } \mathbf{B}_{i k} \text { have the same signs }\right) \\
& \sum_{j \neq i}\left|\frac{\partial \mathbf{h}_{i}}{\partial \mathbf{V}_{j}}\right| \approx \sum_{j \neq i}\left|-\mathbf{B}_{i j}\right|=\sum_{j \neq i}\left|\mathbf{B}_{i j}\right| .
\end{aligned}
$$

So

$$
\left|\frac{\partial \mathbf{g}_{i}}{\partial \theta_{i}}\right| \geq \sum_{j \neq i}\left|\frac{\partial \mathbf{g}_{i}}{\partial \theta_{j}}\right| \quad \text { and } \quad\left|\frac{\partial \mathbf{h}_{i}}{\partial \mathbf{V}_{i}}\right| \geq \sum_{j \neq i}\left|\frac{\partial \mathbf{h}_{i}}{\partial \mathbf{V}_{j}}\right| \text {. }
$$

Therefore, by Gershgorin's theorem [21, p.341], both $\partial \mathrm{g} / \partial \theta$ and $\partial \mathbf{h} / \partial \mathbf{V}$ are nonsingular.
This proves that $\mathbf{J}_{B}^{o}=\partial \mathbf{h} / \partial \mathbf{V}$ is nonsingular. Next consider $\operatorname{det} J_{A}^{\circ}$

$$
\begin{aligned}
\operatorname{det} \mathbf{J}_{A}^{o} & =\operatorname{det}\left[\begin{array}{ccc}
\mathbf{I} & \frac{-\mathbf{S}}{2} \mathbf{I} & \mathbf{O} \\
\frac{\mathbf{s}}{2} \mathbf{M}^{-1} \frac{\partial \mathbf{f}}{\partial \delta} & \mathbf{I}+\frac{\mathbf{s}}{2} \mathbf{M}^{-1} \mathbf{D} & \frac{\mathbf{s}}{2} \mathbf{M}^{-1} \frac{\partial \mathbf{f}}{\partial \theta} \\
\frac{\partial \mathbf{g}}{\partial \delta} & \mathbf{O} & \frac{\partial \mathbf{g}}{\partial \theta}
\end{array}\right] \\
& =\operatorname{det}\left(\frac{\partial \mathbf{g}}{\partial \theta}\right)+\mathbf{O}(\mathbf{s}) .
\end{aligned}
$$

Thus, there exists a small step size $s$ such that $J_{A}^{o}$ is nonsingular. This completes the proof.

Now, we proceed to derive the convergence conditions involved in Step 3). Here, it is convenient to represent the intertive (38)-(39) as follows:

$$
\phi(\mathbf{x})=\mathbf{x}-\mathbf{A}^{-1}[\mathbf{F}(\mathbf{x})]
$$

where

$$
\begin{aligned}
& \phi(\mathbf{x})=\left[\delta_{n}^{k+1}, \omega_{n}^{k+1}, \theta_{n}^{k+1}, \mathbf{V}_{n}^{k+1}\right]^{T} \\
& \mathbf{x}=\left[\delta_{n}^{k}, \omega_{n}^{k}, \theta_{n}^{k}, \mathbf{V}_{n}^{k}\right]^{T} \\
& \mathbf{A}^{-1}=\left[\begin{array}{cc}
\left(\mathbf{J}_{A}^{o}\right)^{-1} & \mathbf{O} \\
\mathbf{O} & \left(\mathbf{J}_{B}^{o}\right)^{-1}
\end{array}\right] \\
& \mathbf{F}(\mathbf{x})=[\Delta(\mathbf{x}), \Omega(\mathbf{x}), \mathbf{G}(\mathbf{x}), \mathbf{H}(\mathbf{x})]^{T} .
\end{aligned}
$$

Then $J_{s}(X)=(\partial F(X) / \partial X)$. Suppose that the successive trajectory point $\left(\delta_{n+1}, \omega_{n+1}, \theta_{n+1}, V_{n+1}\right):=X^{*}$ exists, which is guaranteed by the condition of Lemma 3.1. That is, $F\left(X^{*}\right)=$ $0 \Rightarrow \phi\left(X^{*}\right)=X^{*}$. This implies that $X^{*}$ is a fixed point of the function $\phi(X)$. Suppose that in a neighborhood, $U$, of $X^{*}$ the following condition is satisfied:

$$
\max _{x \in U}\left\|\frac{\partial \phi(\mathbf{x})}{\partial \mathbf{x}}\right\|=\max _{x \in U}\left\|\mathbf{A}^{-1}\left(\mathbf{A}-\mathbf{J}_{s}(\mathbf{x})\right)\right\|=\mathbf{c}<1 .
$$

Then by $C^{1}$ properties of $F\left(X^{*}\right)$ and the mean-value theorem, there exists a ball $B_{\varepsilon}$ which is contained in $U$ and centered at $X^{*}$ with radius $\varepsilon$, i.e.,

$$
\mathbf{B}_{\varepsilon}:=\left\{\mathbf{x}:\left\|\mathbf{x}-\mathbf{x}^{*}\right\| \leq \varepsilon\right\}
$$

such that $\phi(X)$ is a contraction-mapping (in $B_{\varepsilon}$ ) with contraction constant, $c$. Then by the fixed-point tTheorem [19], if the initial point $X^{\circ}$ lies in the $B_{\varepsilon}$ and is sufficiently close to $X^{\circ}$ by the condition $\left\|\phi\left(\mathbf{x}^{o}\right)-\mathrm{x}^{o}\right\|<(1-\mathbf{c}) \varepsilon$, then the sequence $\mathrm{x}^{\circ}, \phi\left(\mathrm{x}^{\circ}\right), \phi\left(\phi\left(\mathrm{x}^{\circ}\right)\right), \ldots$ will converge to the fixed point $\mathbf{x}^{*}=\left(\delta_{n+1}, \omega_{n+1}, \theta_{n+1}, \mathbf{V}_{n+1}\right)$. We summarize the above conditions by the following theorem.

Theorem 3.1 (Sufficient Conditions for Convergence of Step $3)$ : If the following conditions are satisfied:

i) the existence of the successive trajectory point

$$
\left(\delta_{n+1}, \omega_{n+1}, \theta_{n+1}, V_{n+1}\right) \text {; }
$$

ii) $\mathbf{J}_{A}^{0} \& \mathbf{J}_{B}^{0}$ are nonsingular;

iii)

$$
\mathbf{c}=\max _{x \in B_{\varepsilon}}\left\|\mathbf{A}^{-1}\left(\mathbf{A}-\mathbf{J}_{s}(\mathbf{x})\right)\right\|<1 ;
$$

iv) the initial correction, $(\Delta \delta, \Delta \omega, \Delta \theta, \Delta V)$ satisfies

$$
\|(\Delta \delta, \Delta \omega, \Delta \theta, \Delta \mathbf{V})\|<(1-\mathbf{c}) \varepsilon
$$


then the iteration performed in Step 3) converges to the successive trajectory point $\left(\delta_{n+1}, \omega_{n+1}, \theta_{n+1}, \mathbf{V}_{n+1}\right)$ starting from $\left(\delta_{n}, \omega_{n}, \theta_{n}, \mathbf{V}_{n}\right)$.

Remark 3.3: Although Condition iii), which is a complex function of both step size and system structure itself, is difficult to check, our numerical experiments show that most cases will converge by reducing the step size. Thus, in practice, there is no need to directly check Condition iii) before integration. Instead, we put an adaptive step size adjustment procedure in Step 4) of the algorithm in order to obtain convergence.

\section{Piecewise Equivalents Techniques}

Basically, there are two kinds of computations used in piecewise constant current load equivalent (current load equivalent) and piecewise constant transfer admittance equivalent (admittance equivalent), namely, fourth-order Runge-Kutta integration and decoupled power flow calculation.

1) Piecewise Constant Current Load Equivalent (Current Load Equivalent): The fourth-order Runge-Kutta integration is used to integrate the piecewise swing equations (19)-(20). The decoupled power flow method is used for updating equivalent load current injections whenever the generator angles exceed the boundary of the moving hypercube. Since the load bus voltages (magnitudes and angles) do not appear explicitly in the swing equations, they must be updated using a sensitivity analysis.

The load bus voltages can be computed by solving the linearized load flow equations as follows. By linearizing the load flow equations about the base trajectory point centered in the current hypercube, the real and reactive power increments can be expressed in terms of the voltage magnitude increments, the phase angle increments, and the Jacobian matrix $J$. In matrix form we have

$$
\left[\begin{array}{c}
\Delta \mathbf{P}_{g} \\
\Delta \mathbf{P}_{l} \\
\Delta \mathbf{Q}_{g} \\
\Delta \mathbf{Q}_{l}
\end{array}\right]=\left[\begin{array}{llll}
\mathbf{J}_{11} & \mathbf{J}_{12} & \mathbf{J}_{13} & \mathbf{J}_{14} \\
\mathbf{J}_{21} & \mathbf{J}_{22} & \mathbf{J}_{23} & \mathbf{J}_{24} \\
\mathbf{J}_{31} & \mathbf{J}_{32} & \mathbf{J}_{33} & \mathbf{J}_{34} \\
\mathbf{J}_{41} & \mathbf{J}_{42} & \mathbf{J}_{43} & \mathbf{J}_{44}
\end{array}\right]\left[\begin{array}{c}
\Delta \delta_{g} \\
\Delta \delta_{l} \\
\Delta \mathbf{V}_{g} \\
\Delta \mathbf{V}_{l}
\end{array}\right] .
$$

Under the assumptions of constant generator voltage magnitudes $\Delta V_{g}=0$ and ZIP loads, $\Delta P_{l}=\Delta Q_{l}=0$ (40) reduces to

$$
\left[\begin{array}{l}
0 \\
0
\end{array}\right]=\left[\begin{array}{ll}
\mathbf{J}_{22} & \mathbf{J}_{24} \\
\mathbf{J}_{42} & \mathbf{J}_{44}
\end{array}\right]\left[\begin{array}{c}
\Delta \theta_{t} \\
\Delta \mathbf{V}_{l}
\end{array}\right]+\left[\begin{array}{l}
\mathbf{J}_{21} \\
\mathbf{J}_{41}
\end{array}\right] \Delta \delta_{g} .
$$

Rearranging (41) to solve for $\Delta V_{l}$ and $\Delta \theta_{l}$ yields

$$
\left[\begin{array}{c}
\Delta \theta_{l} \\
\Delta \mathbf{V}_{l}
\end{array}\right]=\mathbf{G} \Delta \delta_{g}
$$

where

$$
\mathbf{G}=-\left[\begin{array}{ll}
\mathbf{J}_{22} & \mathbf{J}_{24} \\
\mathbf{J}_{42} & \mathbf{J}_{44}
\end{array}\right]^{-1}\left[\begin{array}{l}
\mathbf{J}_{21} \\
\mathbf{J}_{41}
\end{array}\right]
$$

Since a load flow is performed at each update of load current in the current load equivalent method, the Jacobian matrices in (40) are available for the base trajectory point in the current hypercube. However, it is typical to compute $G$ at the prefault operating point. This does not result in a severe loss of accuracy, whereas the savings in CPU time are considerable. Thus, (42) expresses the load bus voltage magnitude and angle increments as a known function of the generator angle increments. Therefore, the load bus voltage magnitude and angle variations can be monitored in terms of the generator angles within the hypercube.

The current load equivalent technique for real-time transient stability prediction can be summarized as follows.

Step 1) Set up the practical transient security region, $R_{\mathrm{TS}}$ and update parameter $\gamma$. Then compute quantities $Y^{\mathrm{eq}}, D^{\mathrm{eq}}$ and $I_{l}^{o}$ at prefault steady operating condition.

Step 2) Integrate the swing equations [(19) and (20)] and solve the linearized load flow equation [(42)] one step ahead by using arrived phasor measurement. Check whether the one-step prediction value $(\delta(\Delta T), \omega(\Delta T), \theta(\Delta T), V(\Delta T))$ lies within $R_{\mathrm{TS}}$ and predict instability in the case that it does not. If instability has not occurred, the one-step prediction value is checked to see whether it exceeds the boundary of the current hypercube. If it does, recompute the load currents and update the swing equations. Otherwise, no update is made.

Step 3) Repeat Step 2) until either the prediction interval is finished or instability is determined.

2) Piecewise Constant Transfer Admittance Equivalent (Admittance Equivalent): Here, similar to the current load equivalent technique, the fourth-order Runge-Kutta integration is used to integrate the piecewise swing equations ((26) and (27)). The decoupled powerflow is employed to update the transfer admittance matrix whenever the generator angles move beyond the boundary of the current hypercube. The load bus phasor variations are approximated by (42).

The admittance equivalent technique for the real-time transient stability prediction can be summarized as follows.

Step 1) Set up the practical transient security region, $R_{\mathrm{TS}}$ and update parameter $\gamma$. Compute the quantities $Y_{0}^{\text {eq }}, Y_{g l}\left(Y_{l l}-Y_{l}^{o}\right)^{-1}$ and $S$ at the prefault operating point.

Step 2) Integrate the swing equations [(26) and (27)] and solve the linearized load flow equation [(42)] one step ahead. Check whether the one-step prediction value $(\delta(\Delta T), \omega(\Delta T), \theta(\Delta T), V(\Delta T))$ lies within $R_{\mathrm{TS}}$ and predict instability in the case that it does not. If instability has not occurred, the one-step prediction value is checked to see whether it exceeds the boundary of the current hypercube. If it does, recompute the transfer admittances and update the swing equations. Otherwise, the transfer admittances remain unchanged.

Step 3) Repeat Step 2) until either the prediction interval of interest is finished or else instability is determined.

\section{NUMERICAL EXPERIMENTS}

This section presents numerical simulations of the proposed techniques, i.e., decoupled integration, current load equivalent and admittance equivalent, on two sample power systems. The first system is a four-machine six-bus system with ZIP load 


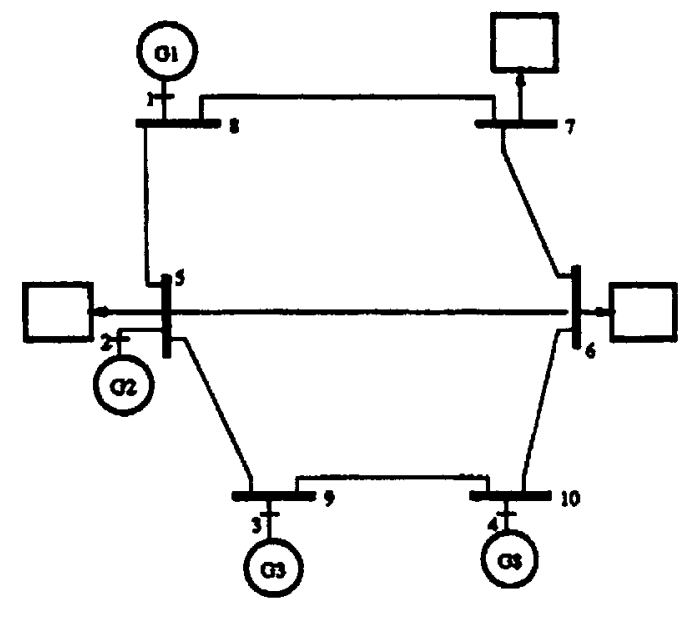

PQ Load

: Generator

\section{4-Machine 3-PQ Load Power System}

Fig. 5. One-line diagram of the four-machine system.

TABLE I

Average CPU TIME (SECONDS) OF THE DECOUPLED INTEGRATION TECHNIQUE AND THE FULL JACOBIAN METHOD FOR THE FouR-Machine SySTEM 1-S DYNAMIC RESPONSE

\begin{tabular}{c|c|c}
\hline Disturbance & Decoupled Integration & Full Jacobian \\
\hline Stable Swing & 0.18 & 0.64 \\
\hline Unstable Swing & 0.20 & 0.67 \\
\hline
\end{tabular}

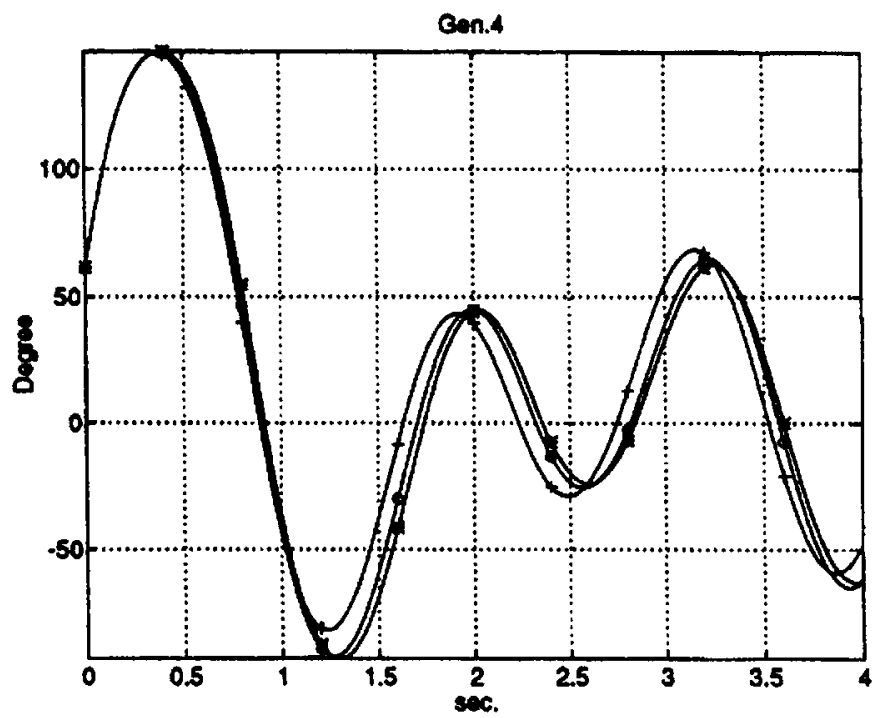

Fig. 6. Representative stable angle swing of Gen. 4 calculated by decoupled integration and current load equivalent using step size, $0.02 \mathrm{~s} *$ : decoupled integration, $\mathrm{CPU}=0.7 \mathrm{~s}+$ : current load equivalent $(\gamma=\infty), \mathrm{CPU}=0.11$ s. $o$ : current load equivalent $\left(\gamma=60^{\circ}\right), \mathrm{CPU}=0.16 \mathrm{~s}$.

$(a=1, b=0, c=0, d=1, e=0, f=0)$, taken from a seminal paper on transient stability regions [17]. The second sample power system is the New England ten-machine, 39-bus system with ZIP load $(a=0.7, b=0.1, c=0.2, d=0.7, e=0.1, f=$ 0.2 ). The programs are coded in FORTRAN and implemented

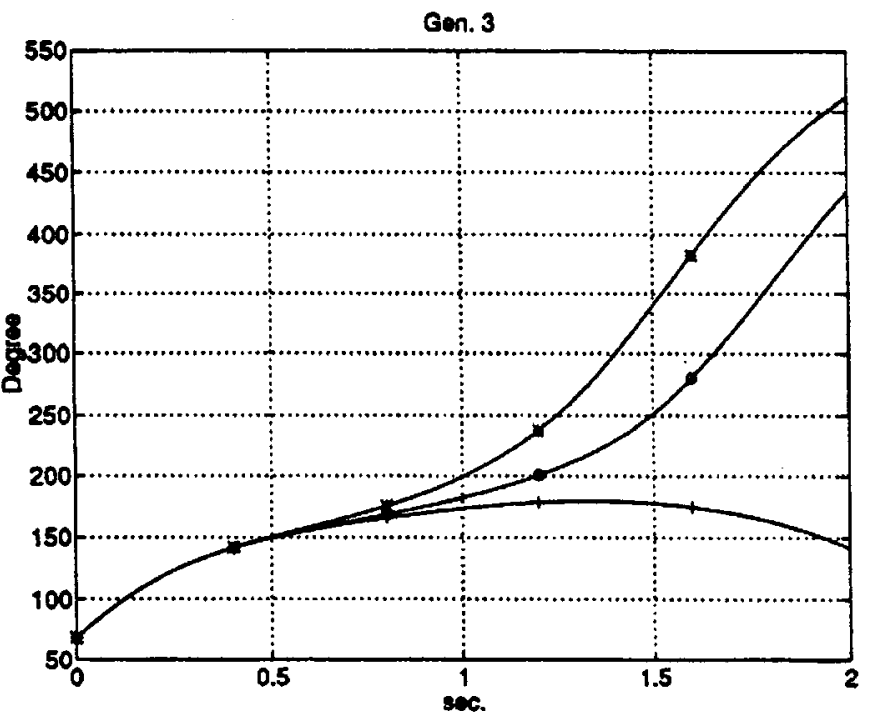

Fig. 7. Representative unstable angle swing of Gen. 4 calculated by decoupled integration and current load equivalent using step size, $0.02 \mathrm{~s} *$ : decoupled integraion, $\mathrm{CPU}=0.55 \mathrm{~s}+$ : current load equivalent $(\gamma=\infty), \mathrm{CPU}=0.05$ so: current load equivalent $\left(\gamma=60^{\circ}\right), \mathrm{CPU}=0.07 \mathrm{~s}$

on an HP 9000/720 workstation. There are two important metrics, CPU time and approximation error, for evaluating the effectiveness of the proposed techniques for the real-time prediction problem. The CPU time was measured by placing timing routine calls within the program. The first call was placed before the postfault computation (not including the computation of the prefault quantities such as $J_{A}^{o}, J_{B}^{o}, Y^{\mathrm{eq}}, D^{\mathrm{eq}}, I_{l}\left(\delta_{o}\right), Y_{o}^{\mathrm{eq}}, S$ and $Y_{g l}\left(Y_{l l}-Y_{l}^{o}\right)^{-1}$ and the second call was placed at the end. The approximation errors were measured by the following formula:

$$
\mathbf{E}=\max _{k \in \text { preditctional interval }}\|\delta(\mathbf{k} \Delta \mathbf{T})-\bar{\delta}(\mathbf{k} \Delta \mathbf{T})\|_{\infty}
$$

where

$\delta(\mathbf{k} \Delta \mathbf{T}) \quad$ generator angle calculated by proposed techniques; 


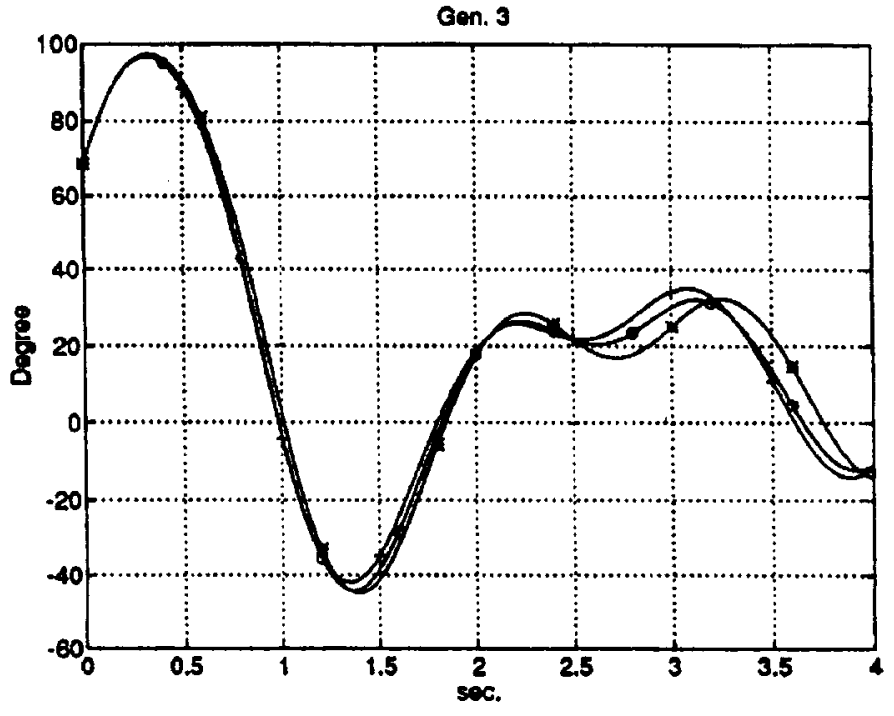

Fig. 8. Representative stable angle swing of Gen. 3 calculated by decoupled integration and admittance equivalent using step size, $0.02 \mathrm{~s} *$ : decoupled integration, $\mathrm{CPU}=0.7 \mathrm{~s} .+$ : admittance equivalent $(\gamma=\infty), \mathrm{CPU}=0.10$ $\mathrm{s} \circ$ : admittance equivalent $\left(\gamma=60^{\circ}\right), \mathrm{CPU}=0.13 \mathrm{~s}$

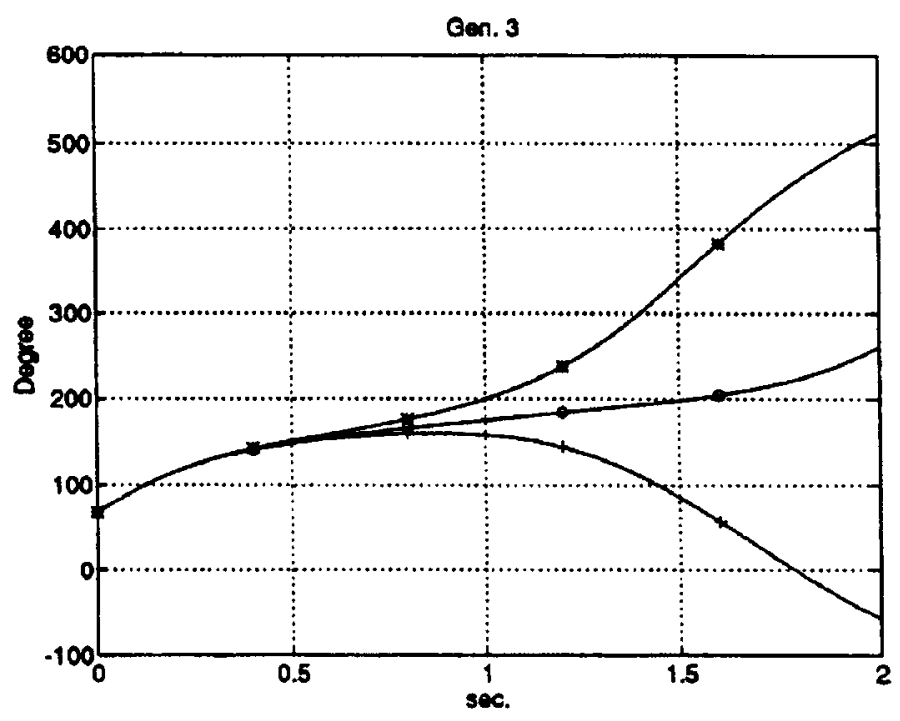

Fig. 9. Representative unstable angle swing of Gen. 3 calculated by decoupled integration and admittance equicalent using step size, $0.02 \mathrm{~s} *$ : decoupled integration, $\mathrm{CPU}=0.55 \mathrm{~s}+$ : admittance equivalent $(\gamma=\infty), \mathrm{CPU}=0.05$ so: admittance equivalent $\left(\gamma=60^{\circ}\right), \mathrm{CPU}=0.06 \mathrm{~s}$

$\bar{\delta}(\mathbf{k} \Delta \mathbf{T}) \quad$ generator angle calculated by ETMSP simulation package [20];

$\|\cdot\|_{\infty}: \quad l$-infinite norm.

Note that we regarded the generator angles calculated by the ETMSP as the accurate values compared to generator angles calculated by the piecewise equivalent techniques. The justification is obvious because the ETMSP did not make any approximations in the adopted zip load model.

1) Test System 1: The four-machine six-bus system is shown in Fig. 5. We treated Gen. 1 of the four -machine system as the slack bus in the initialization since it had the largest inertia constant. Three-phase short circuit to ground faults were simulated to occur on various lines with two-cycle (1/30 s) fault clearing times. In the first phase of the simulation, we timed the speed of

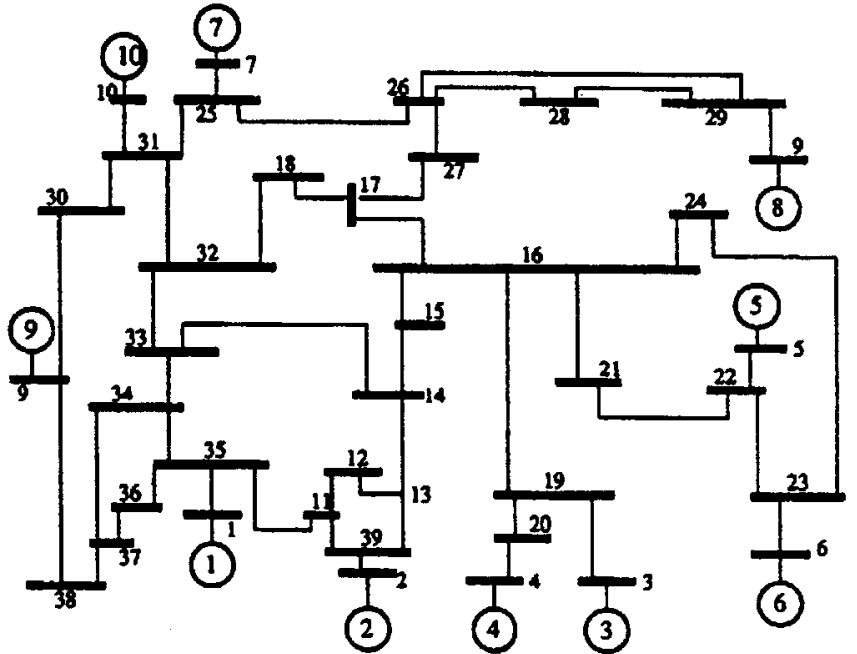

Fig. 10. One-line diagram of ten-machine system.

the decoupled integration technique when calculating stable and unstable swings caused by different fault locations. For comparison, we also measured the speed of integrating the same stable and unstable swings by implicit trapezoidal integration with a full Jacobian update at every time step and the same stop criteria as decoupled integration technique. This is based on the fact that the decoupled integration technique has the same accuracy as the full Jacobian method. The average timing results are shown in Table I, showing that the decoupled integration technique is about three-four times faster than the integration technique which uses a full Jacobian update at every time step. It is observed that the integration speed of the decoupled integration technique is faster than the real power system response time (1 s) for both stable and unstable swings. Thus, the decoupled integration technique can predict the stability of swings in real time for the four-machine system.

In the second phase of simulation, we compared the performance of the decoupled integration technique and the two piecewise equivalent techniques, current load equivalent, and admittance equivalent by timing the CPU time and measuring the approximation errors. A representative $4 \mathrm{~s}$ stable transient swing was integrated by the decoupled integration and current load equivalent techniques using both no update $(\gamma=\infty)$ and $60^{\circ}$ update $\left(\gamma=60^{\circ}\right)$ for the current load equivalent method. The corresponding swing curves computed for Gen. 4 are shown in Fig. 6. The CPU times are $0.7 \mathrm{~s}$ for the decoupled integration, $0.11 \mathrm{~s}$ for the current load equivalent $(\gamma=\infty)$, and $0.16 \mathrm{~s}$ for the current load equivalent $\left(\gamma=60^{\circ}\right)$. The approximation errors for the current load equivalent $(\gamma=\infty)$ and current load equivalent $\left(\gamma=60^{\circ}\right)$ are $52^{\circ}$ and $21^{\circ}$, respectively. Note that all three integration techniques can predict the swing in real time for this case. Moreover, it only takes $0.05 \mathrm{~s}$ more CPU time for the current load equivalent $\left(\gamma=60^{\circ}\right)$ to reduce the approximation errors by about $60 \%$ from the current load equivalent $(\gamma=\infty)$. In Fig. 7, a representative $2 \mathrm{~s}$ unstable swing is shown for Gen. 3 . The CPU time of this case are $0.55 \mathrm{~s}$ for the decoupled integration, 0.05 sfor the current load equivalent $(\gamma=\infty)$ and 0.07 $\mathrm{s}$ for the current load equivalent $\left(\gamma=60^{\circ}\right)$. This case is especially significant since the current load equivalent $(\gamma=\infty)$ 
TABLE II

CPU TIME OF VARIOUS TECHNIQUES FOR 1-S DYNAMIC RESPONSE OF A 39-BUS SYSTEM

\begin{tabular}{c|c|c|c|c|c}
\hline Fault Loc. & $\begin{array}{c}\text { Decoupled } \\
\text { Integration }\end{array}$ & \multicolumn{2}{|c|}{ Current Load Equivalent } & \multicolumn{2}{|c}{ Admittance Equivalent } \\
\hline Bus-Bus & $\begin{array}{c}\text { CPU Time } \\
\text { (sec.) }\end{array}$ & \multicolumn{2}{|c|}{ CPU Time (sec.) } & \multicolumn{2}{c}{ CPU Time (sec.) } \\
\cline { 3 - 6 } & $\gamma=5^{\circ}$ & $\gamma=10^{\circ}$ & $\gamma=10^{\circ}$ & $\gamma=30^{\circ}$ \\
\hline $11-12$ & 2.71 & 0.51 & 0.44 & 0.75 & 0.32 \\
\hline $13-14$ & 2.75 & 0.54 & 0.50 & 0.76 & 0.55 \\
\hline $15-16$ & 2.66 & 0.56 & 0.51 & 0.73 & 0.54 \\
\hline $33-34$ & 3.27 & 0.63 & 0.49 & 0.90 & 0.50 \\
\hline $36-37$ & 3.58 & 0.62 & 0.50 & 0.89 & 0.52 \\
\hline
\end{tabular}

TABLE III

ERRORS OF PROPOSED PIECEWISE EQUIVALENT TECHNIQUES FOR 1-S DYNAMIC RESPONSE OF A 39-Bus SYSTEM

\begin{tabular}{c|c|c|c|c}
\hline Fault Location. & \multicolumn{2}{|c|}{ Current Load Equivalent } & \multicolumn{2}{c}{ Admittance Equivalent } \\
\hline \multirow{2}{*}{ Bus-Bus } & \multicolumn{2}{|c|}{ Error(deg.) } & \multicolumn{2}{c}{ Error(deg.) } \\
\cline { 2 - 5 } & $\gamma=5^{\circ}$ & $\gamma=10^{\circ}$ & $\gamma=10^{\circ}$ & $\gamma=30^{\circ}$ \\
\hline $11-12$ & 8.57 & 15.52 & 9.98 & 20.61 \\
\hline $13-14$ & 8.19 & 17.80 & 6.94 & 12.01 \\
\hline $15-16$ & 4.90 & 12.83 & 5.34 & 9.29 \\
\hline $33-34$ & 6.62 & 15.87 & 7.82 & 8.70 \\
\hline $36-37$ & 2.87 & 5.99 & 5.56 & 8.68 \\
\hline
\end{tabular}

calculation, which represents the typical constant current load model, yields the wrong stability prediction, while the current load equivalent $\left(\gamma=60^{\circ}\right)$ takes only $0.02 \mathrm{~s}$ additional CPU time and predicts thesame instability as the decoupled integration.

We conducted similar simulations using transfer admittance both without update admittance equivalent $(\gamma=\infty)$ and with $60^{\circ}$ updates admittance equivalent $\left(\gamma=60^{\circ}\right)$. The results are shown in Figs. 8 and 9. Again, it is significant that for the unstable case the admittance equivalent $(\gamma=\infty)$ predicts the wrong stability status while admittance equivalent $\left(\gamma=60^{\circ}\right)$ takes only $0.01 \mathrm{~s}$ additional CPU time to give same results as the decoupled integration.

2) Test System 2: The IEEE ten-machine 39-bus system is also used to illustrate the performance of our integration techniques. The one-line diagram of this system is shown in Fig. 10. Although this system is not very large, it is a good example of a reduced-order system in an interconnected network, representing the $345-\mathrm{kV}$ transmission network of New England [18].

Three-phase short circuits to ground faults were simulated to occur on various transmission lines. The fault duration was two cycles, followed by tripping the appropriate circuit breakers. The postfault system configuration is the same as the prefault system, except that the faulted line is eliminated. We timed the execution time for the calculation of $1 \mathrm{~s}$ of postfault dynamic response and measured the approximation errors. Each technique was tested with different step sizes on an HP 9000/720 workstation. The representative simulation results are shown in Tables II and III, where it can be seen that both current load equivalent and admittance equivalent can predict the swings in real time with an acceptable degree of accuracy. For example, the approximation errors of current load equivalent $\left(\gamma=5^{\circ}\right)$ and admittance equivalent $\left(\gamma=10^{\circ}\right)$ with step size $(0.02 \mathrm{~s})$ are less than $10^{\circ}$. Although decoupled integration can not be faster than real-time response for this test system, it would be possibe to speed up decoupled integration to achieve real-time prediction by upgrading the workstation used for simulation. By increasing the step size, we achieved a faster calculation, although the approximation errors became larger. Moreover, in this test system we found that the decoupled integration would diverge if we used a step size larger than $0.06 \mathrm{~s}$ for most fault locations.

\section{CONCLUDING REMARKS}

Decoupled integration, current load equivalent and admittance equivalent techniques were proposed and tested on two sample power systems to show solutions for a real-time transient stability prediction problem. Depending on the nature of the system, one of these three techniques may be selected to provide increased speed as well as accuracy. If there are several computers connected by network in the control center, then the proposed three techniques can be sped up by paralleling the algorithms. Since the update matrices $J_{A}^{o}$ and $J_{B}^{o}$ are constant in decoupled integration, decoupled integration is inherently parallel. For the two piecewise techniques, parallel Runge-Kutta schemes are already available from the literature. Therefore, greatly improved performance of computer-based transient stability prediction can be expected in actual situations by using the proposed techniques.

\section{REFERENCES}

[1] J. S. Thorp, A. G. Phadke, S. H. Horowitz, and M. M. Begovic, "Some applications of phasor measurements to adaptive protection," IEEE Trans. Power Syst., vol. 3, pp. 791-798, May 1988.

[2] J. S. Thorp, "Control of electric power system using real-time measurement," IEEE Contr. Syst. Mag., pp. 39-45, Jan. 1989.

[3] A. G. Phadke and J. S. Thorp, Computer Relaying for Power Systems: Research Studies, 1988.

[4] A. G. Phadke, J. S. Thorp, and M. G. Adamiak, "A new measurement technique for tracking voltage phasor, local system frequency and rate of change of frequency," IEEE Trans. Power Apparatus Syst., vol. 102, pp. 1025-1038, May 1983.

[5] A. G. Phadke, "Synchronized phasor measurements in power systems," IEEE Comput. Appl. Power, vol. 6, pp. 10-15, 1993.

[6] R. P. Schulz, L. S. Van Slyck, and S. H. Horowitz, "Application of fast phasor measurements on utility systems," in Proc. PICA, May 1989, pp. $49-55$.

[7] A. G. Phadke and J. S. Thorp, "Improved control and protection of power systems though synchronized phasor measurements," in Control and Dynamic Systems. New York: Academic , 1991, vol. 43, pp. 335-376.

[8] V. Centeno et al., "Adaptive out-of-step relaying using phasor measurement techniques," IEEE Comput. Appl. Power, vol. 6, pp. 12-17, 1993.

[9] S. Rovnyak, S. Kretsinger, J. S. Thorp, and D. Brown, "Decision trees for real-time transient stability prediction," IEEE Trans. Power Syst., vol. 9, pp. 1417-1426, Aug. 1994.

[10] S. Rovnyak, C.-W. Liu, J. Lu, W. Ma, and J. S. Thorp, "Predicting future behavior of transient events rapidly enough to evaluate remedial control options in real-time," IEEE Trans. Power Syst., vol. 10, pp. 1195-1203, 1995.

[11] C.-W. Liu, M.-C. Su, S.-S. Tsay, and Y.-J. Wang, "Application of a novel fuzzy neural network to real-time transient stability swing prediction based on synchronized phasor measurements," IEEE Trans. Power Syst., vol. 14, pp. 685-692, May 1999.

[12] M. H. Kent et al., "Dynamic modeling of loads in stability studies," IEEE Trans. Power Apparatus Syst., vol. 88, pp. 756-763, May 1969.

[13] presented at the 1988 System Disturbances, July 1989. 
[14] T. Baldwin, L. Mili, and A. G. Phadke, "Dynamic ward equivalents for transient stability analysis," IEEE Trans. Power Syst., vol. 9, pp. 59-67, 1994.

[15] C. W. Gear, "Simultaneous numerical solution of differential-algebraic equations," IEEE Trans. Circuit Theory, vol. CT-18, Jan. 1971.

[16] H. W. Dommel and N. Sato, "Fast transient stability solution," IEEE Trans. Power Apparatus Syst., vol. PAS-91, pp. 1643-1650, July/Aug. 1972.

[17] A. H. El-Abiad and K. Nagappan, "Transient stability region of multi-machine power systems," IEEE Trans. Power Apparatus Syst., vol. PAS-85, no. 2, pp. 169-178, Feb. 1966.

[18] M. H. Haque and A. H. M. A. Rahim, "Determination of first swing stability limit of multimachine power systems through taylor series expansion," Proc. Inst. Elect. Eng., pt. C, vol. 136, no. 6, pp. 373-379, 1989.

[19] J. M. Ortega and W. C. Rheinboldt, Iterative Solution of Nonlinear Equations in Several Variables. New York: Academic , 1970.

[20] "Extended Transient/Midterm Stability Program," EPRI, Ontario Hydro, TR-102004-V2R1, Version 3.1, May 1994.

[21] G. H. Golub and C. F. Van Loan, Matrix Computation. Baltimore, MD: The Johns Hopkins University Press, 1989.
Chih-Wen Liu (S'93-M'96) was born in Taiwan in 1964. He received the B.S degree in electrical engineering from National Taiwan University, Taipei, in 1987 and the Ph.D. degree in electrical engineering from Cornell University, Ithaca, NY, in 1994.

Since 1994, he has been with National Taiwan University, where he is an Associate Professor of electrical engineering. His research interests include power system computer applications.

James S. Thorp (S'58-M'63-SM'80-F'89) received the B.E.E., M.S., and $\mathrm{Ph} . \mathrm{D}$. degrees from Cornell University, Ithaca, NY.

He joined the faculty of Cornell in 1962, where he is currently the Charles N. Mellowes Professor in Engineering and Director of the School of Electrical Engineering. In 1976, he was a Faculty Intern with the AEP Service Corporation. In 1988, he was an Overseas Fellow at Churchill College, Cambridge, U.K.

Dr. Thorp was an Associate Editor of the IEEE TRANSACTIONS ON CIRCUIT AND SYSTEMS from 1985 to 1987. He is a member of the National Academy of Engineering and is member of the IEEE Power System Relaying Committee, CIGRE, Eta Kappa Nu, Tau Beta Pi, and Sigma Xi. 